\title{
Heavy Higgs decays into sfermions in the complex MSSM: a full one-loop analysis
}

\author{
S. Heinemeyer ${ }^{1, \mathrm{a}}$, C. Schappacher ${ }^{2, \mathrm{~b}}$ \\ ${ }^{1}$ Instituto de Física de Cantabria (CSIC-UC), Santander, Spain \\ ${ }^{2}$ Institut für Theoretische Physik, Karlsruhe Institute of Technology, 76128 Karlsruhe, Germany
}

Received: 24 October 2014 / Accepted: 31 March 2015 / Published online: 7 May 2015

(C) The Author(s) 2015. This article is published with open access at Springerlink.com

\begin{abstract}
For the search for additional Higgs bosons in the Minimal Supersymmetric Standard Model (MSSM) as well as for future precision analyses in the Higgs sector a precise knowledge of their decay properties is mandatory. We evaluate all two-body decay modes of the heavy Higgs bosons into sfermions in the MSSM with complex parameters (cMSSM). The evaluation is based on a full one-loop calculation of all decay channels, also including hard QED and QCD radiation. The dependence of the heavy Higgs bosons on the relevant cMSSM parameters is analyzed numerically. We find sizable contributions to many partial decay widths. They are roughly of $\mathcal{O}(15 \%)$ of the tree-level results, but can go up to $30 \%$ or higher. The size of the electroweak one-loop corrections can be as large as the QCD corrections. The full one-loop contributions are important for the correct interpretation of heavy Higgs-boson search results at the LHC and, if kinematically allowed, at a future linear $e^{+} e^{-}$collider. The evaluation of the branching ratios of the heavy Higgs bosons will be implemented into the Fortran code FeynHiggs.
\end{abstract}

\section{Introduction}

One of the most important tasks at the LHC is to search for physics effects beyond the Standard Model (SM), where the Minimal Supersymmetric Standard Model (MSSM) [1-4] is one of the leading candidates. Supersymmetry (SUSY) predicts two scalar partners for all SM fermions as well as fermionic partners to all SM bosons. Another important task is investigating the mechanism of electroweak symmetry breaking. The most frequently investigated models are the Higgs mechanism within the SM and within the MSSM.

C. Schappacher was formerly affiliated with Institut für Theoretische Physik, Karlsruhe Institute of Technology.

\footnotetext{
a e-mail: Sven.Heinemeyer@cern.ch

b e-mail: schappacher@kabelbw.de
}

Contrary to the case of the SM, in the MSSM two Higgs doublets are required. This results in five physical Higgs bosons instead of the single Higgs boson in the SM; three neutral Higgs bosons, $h_{n}(n=1,2,3)$, and two charged Higgs bosons, $H^{ \pm}$. The Higgs sector is described at the tree level by two parameters: the mass of the charged Higgs boson, $M_{H^{ \pm}}$, and the ratio of the two vacuum expectation values, $\tan \beta \equiv t_{\beta}=v_{2} / v_{1}$. Often the lightest Higgs boson, $h_{1}$ is identified with the particle discovered at the LHC $[5,6]$ with a mass around $\sim 125 \mathrm{GeV}$. If the mass of the charged Higgs boson is assumed to be larger than $\sim 200 \mathrm{GeV}$ the four additional Higgs bosons are roughly mass degenerate, $M_{H^{ \pm}} \approx m_{h_{2}} \approx m_{h_{3}}$, and they are referred to as the "heavy Higgs bosons". Discovering one or more of those additional Higgs bosons would be an unambiguous sign of physics beyond the SM and could yield important information as regards their supersymmetric origin.

If SUSY is realized in nature and the charged Higgs-boson mass is $M_{H^{ \pm}} \lesssim 1.5 \mathrm{TeV}$, then the heavy Higgs bosons could be detectable at the LHC $[7,8]$ (including its high luminosity upgrade, HL-LHC; see Ref. [9] and references therein) and/or at a future linear $e^{+} e^{-}$collider such as the ILC [10-14] or CLIC $[15,16]$. (Results on the combination of LHC and LC results can be found in Refs. [17-19].) The discovery potential at the HL-LHC goes up to $\mathcal{O}(1 \mathrm{TeV})$ for large $\tan \beta$ values and somewhat lower at low $\tan \beta$ values. At an $e^{+} e^{-}$linear collider the heavy Higgs bosons are pair produced, and the reach is limited by the center-of-mass energy, $M_{H^{ \pm}} \lesssim \sqrt{s} / 2$, roughly independent of $\tan \beta$. Details as regards the discovery process(es) depend strongly on the cMSSM parameters (and will not be further discussed in this paper).

In the case of a discovery of additional Higgs bosons a subsequent precision determination of their properties will be crucial to determine their nature and the underlying (SUSY) parameters. In order to yield a sufficient accuracy, one-loop corrections to the various Higgs-boson decay modes have to be considered. Decays to SM fermions have been evaluated 
at the full one-loop level in the cMSSM in Ref. [20]; see also Refs. [21] as well as Refs. [22-28] for higher-order SUSY corrections. Decays to (lighter) Higgs bosons have been evaluated at the full one-loop level in the cMSSM in Ref. [20]; see also Ref. [29]. Decays to SM gauge bosons can be evaluated to a very high precision using the full SM one-loop result [30-32] combined with the appropriate effective couplings [33]. The full one-loop corrections in the cMSSM listed here together with resummed SUSY corrections have been implemented into the code FeynHiggs [33-38]. Corrections at and beyond the one-loop level in the MSSM with real parameters (rMSSM) are implemented into the code HDECAY [3941]. Both codes were combined by the LHC Higgs Cross Section Working Group to obtain the most precise evaluation for rMSSM Higgs-boson decays to SM particles and decays to lighter Higgs bosons [42].

The heavy MSSM Higgs bosons can also decay to SUSY particles, i.e. to charginos, neutralinos, and scalar fermions. In Ref. [43] it was demonstrated that the SUSY particle modes can dominate the decay of the heavy Higgs bosons. In this work we calculate all two-body decay modes of the heavy Higgs bosons to scalar fermions in the cMSSM. ${ }^{1}$ More specifically, we calculate the full one-loop corrections to the partial decay widths

$\Gamma\left(h_{n} \rightarrow \tilde{f}_{i} \tilde{f}_{j}^{\dagger}\right) \quad(n=2,3 ; i, j=1,2)$,

$\Gamma\left(H^{+} \rightarrow \tilde{f}_{i} \tilde{f}_{j}^{\prime \dagger}\right)$ and $\Gamma\left(H^{-} \rightarrow \tilde{f}_{i}^{\dagger} \tilde{f}_{j}^{\prime}\right) \quad(i, j=1,2)$,

where $H^{ \pm}$denotes the charged, $h_{n}$ the mixed neutral Higgs bosons and $\tilde{f}\left(\tilde{f}^{\dagger}\right)$ denotes the scalar (anti-) fermions. ${ }^{2}$ The total decay width is defined as the sum of the partial decay widths (1) or (2), the SM decay channels as described above and the decays to charginos/neutralinos (at the tree level, supplemented with effective couplings [33]).

The evaluation of the channels Eqs. (1), (2) is based on a full one-loop calculation, i.e. including (S)QCD and electroweak (EW) corrections, as well as soft and hard QCD and QED radiation. For "mixed" decay modes, we evaluate in addition the two "CP $\mathcal{P}$-versions" $(i \neq j)$ of Eq. (1) and the two "CP -versions" of Eq. (2), which give different results for non-zero complex phases. While our calculation comprises the decay to all sfermionic decay modes of the cMSSM Higgs bosons, in our numerical analysis we will focus on the decay to the third generation sfermions, scalar top and bottom quarks, scalar tau and tau neutrinos.

Higher-order contributions to MSSM Higgs decays to scalar fermions have been evaluated in various analyses over the last decade. However, they were in most cases restricted

\footnotetext{
1 We neglect flavor violation effects and resulting decay channels.

2 In the text and figures below we omit the ${ }^{\dagger}$ (denoting anti-particles) for simplification.
}

to a few specific channels. In many cases only parts of a oneloop calculation has been performed, and no higher-order corrections in the cMSSM are available so far. More specifically, the available literature comprises the following. First, $\mathcal{O}\left(\alpha_{s}\right)$ corrections to partial decay widths of various squark decay channels in the rMSSM were derived: to the decay of a charged Higgs to stops and sbottoms in Ref. [44], of a heavy Higgs boson to third generation squarks in Ref. [45], supplemented later by an effective resummation of the trilinear Higgs-sbottom coupling in Ref. [46]. First full one-loop corrections in the rMSSM were calculated in the decays of the $\mathcal{C P}$-odd Higgs boson to scalar quarks [47] and to scalar fermions [48]. The full one-loop corrections in the rMSSM to Higgs decays to squarks was published in Ref. [49]. More recently, the results of this group were made public in the code HFOLD [50], using a pure $\overline{\mathrm{DR}}$ renormalization for the calculation. While their results constitute a full one-loop correction (although not for complex parameters), it differs from our calculation in the renormalization of the SUSY particles and parameters. It was shown in Refs. [51-54] that our renormalization leads to stable results over nearly the full cMSSM parameters space.

The full $\mathcal{O}\left(\alpha_{s}\right)$ corrections to Higgs decays to scalar quarks were also evaluated by a different group in Ref. [55], using a renormalization more similar to ours, but also restricting to the case of real parameters. Finally, in Ref. [56] the $\mathcal{O}\left(\alpha_{s}\right)$ corrections to Higgs decays to scalar quarks were re-analyzed, where the emphasis was put on the connection of the MSSM squark sector and Higgs sector couplings to $\overline{\mathrm{MS}}$ input parameters. The latter corrections in particular differ from our treatment of the renormalization of the scalar quark sector. They have been included into the code HDECAY.

In this paper we present for the first time a full one-loop calculation for all two-body sfermionic decay channels of the Higgs bosons in the cMSSM (with no generation mixing), taking into account soft and hard QED and QCD radiation. In Sect. 2 we review the renormalization of all relevant sectors of the cMSSM. Details about the calculation can be found in Sect. 3, and the numerical results for all decay channels are presented in Sect. 4 (including comments on comparisons with results from other groups). The conclusions can be found in Sect. 5. The results will be implemented into the Fortran code FeynHiggs [33-38].

\section{The complex MSSM}

The channels (1) and (2) are calculated at the one-loop level, including hard QED and QCD radiation. This requires the simultaneous renormalization of several sectors of the cMSSM, including the colored sector with top and bottom quarks and their scalar partners and the Higgs and gaugeboson sector with all the Higgs bosons as well as the $Z$ and 
the $W$ boson and the chargino/neutralino sector. In the following subsections we briefly review these sectors and their renormalization.

\subsection{The Higgs- and gauge-boson sector}

The Higgs- and gauge-boson sector follow strictly Ref. [57] and references therein (see especially Ref. [33]). This defines in particular the counterterm $\delta \tan \beta \equiv \delta t_{\beta}$, as well as the counterterms for the $Z$ boson mass, $\delta M_{Z}^{2}$, and the sine of the weak mixing angle, $\delta s_{\mathrm{w}}$.

\subsection{The chargino/neutralino sector}

The chargino/neutralino sector is also described in detail in Ref. [57] and references therein. In this paper we use the so called CCN scheme, i.e. on-shell conditions for two charginos and one neutralino, which we chose to be the lightest one. In the notation of Ref. [57] we used:

\$InoScheme $=$ CCN[1] - fixed CCN scheme with on-shell $\tilde{\chi}_{1}^{0}$.

This defines in particular the counterterm $\delta \mu$, where $\mu$ denotes the Higgs mixing parameter.

\subsection{The fermion sector}

The fermion sector is described in detail in Ref. [57] and references therein. For simplification we use here the $\overline{\mathrm{DR}}$ renormalization for all three generations of down-type quarks and leptons, again in the notation of Ref. [57]:

UVMf1 [4,_] = UVDivergentPart

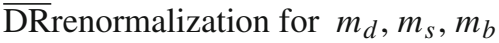

UVMf1 [2,_] = UVDivergentPart

$\overline{\mathrm{DR}}$ renormalization for $m_{e}, m_{\mu}, m_{\tau}$

\subsection{The scalar fermion sector}

The renormalization of the sfermion sector which we use here differs slightly from the one described in Ref. [57]. For the renormalization of the squark sector we follow $\mathcal{O} 2$ in Refs. [51,52] (see also Ref. [53]) and for the slepton sector we created an additional $\overline{\mathrm{DR}}$ type version in full analogy to the squark sector. ${ }^{3}$ In the following we list these new formulas we used in this analysis.

In the absence of non-minimal flavor violation, the sfermion mass matrix is given by $[3,4]$

$\mathbf{M}_{\tilde{f} t g}^{2}=\left(\begin{array}{cc}\left(\mathbf{M}_{L, f_{t}}^{2}\right)_{g g}+m_{f_{t g}}^{2} & m_{f_{t g}}\left(\mathbf{X}_{f_{t}}\right)_{g g}^{*} \\ m_{f_{t g}}\left(\mathbf{X}_{f_{t}}\right)_{g g} & \left(\mathbf{M}_{R, f_{t}}^{2}\right)_{g g}+m_{f_{t g}}^{2}\end{array}\right)$

3 The main difference between the renormalization in Ref. [57] and the one used in this paper is that we impose a further on-shell renormalization condition for the $\mathrm{d}_{g}$ - and $\mathrm{e}_{g}$-type sfermion masses, including an explicit restoration of the $S U(2)_{L}$ relation. where

$$
\begin{aligned}
\mathbf{M}_{L, f_{t}}^{2}= & M_{Z}^{2}\left(I_{3}^{f_{t}}-Q_{f_{t}} s_{\mathrm{w}}^{2}\right) c_{2 \beta} \\
& + \begin{cases}\mathbf{M}_{\tilde{L}}^{2} & \text { for left-handed sleptons }(t=1,2), \\
\mathbf{M}_{\tilde{Q}}^{2} & \text { for left-handed squarks }(t=3,4),\end{cases} \\
\mathbf{M}_{R, f_{t}}^{2}= & M_{Z}^{2} Q_{f_{t}} s_{\mathrm{w}}^{2} c_{2 \beta} \\
& + \begin{cases}\mathbf{M}_{\tilde{E}}^{2} & \text { for right-handed sleptons }(t=2), \\
\mathbf{M}_{\tilde{U}}^{2} & \text { for right-handed } u \text {-type squarks }(t=3), \\
\mathbf{M}_{\tilde{D}}^{2} & \text { for right-handed } d \text {-type squarks }(t=4),\end{cases} \\
& \times \begin{cases}1 / t_{\beta} & \text { for isospin-up sfermions }(t=3), \\
t_{\beta} & \text { for isospin-down sfermions }(t=2,4),\end{cases}
\end{aligned}
$$

The soft-SUSY-breaking parameters $\mathbf{M}_{\tilde{L}, \tilde{Q}, \tilde{E}, \tilde{U}, \tilde{D}}^{2}$ and $\mathbf{A}_{f}$ are $3 \times 3$ matrices in flavor space whose off-diagonal entries are zero in the minimally flavor-violating MSSM. $Q_{f}$ and $I_{3}^{f}$ denote the charge and the weak iso-spin of the corresponding fermion, and $c_{2 \beta} \equiv \cos 2 \beta$.

The mass matrix is diagonalized by a unitary transformation $\mathbf{U}_{\tilde{f}}$,

$$
\mathbf{U}_{\tilde{f}} \mathbf{M}_{\tilde{f}}^{2} \mathbf{U}_{\tilde{f}}^{\dagger}=\left(\begin{array}{cc}
m_{\tilde{f}_{1}}^{2} & 0 \\
0 & m_{\tilde{f}_{2}}^{2}
\end{array}\right), \quad \mathbf{U}_{\tilde{f}}=\left(\begin{array}{cc}
U_{11}^{\tilde{f}} & U_{12}^{\tilde{f}} \\
U_{21}^{\tilde{f}} & U_{22}^{\tilde{f}}
\end{array}\right) .
$$

We renormalize the up-type squarks $\left(\tilde{\mathrm{u}}_{\{g=1,2,3\}}=\{\tilde{u}, \tilde{c}, \tilde{t}\}\right)$ and the sneutrinos $\left(\tilde{v}_{\{g=1,2,3\}}=\left\{\tilde{v}_{e}, \tilde{v}_{\mu}, \tilde{v}_{\tau}\right\}\right)$ on-shell (OS). For the down-type squarks $\left(\tilde{\mathrm{d}}_{\{g=1,2,3\}}=\{\tilde{d}, \tilde{s}, \tilde{b}\}\right)$ and the electron-type sleptons $\left(\tilde{\mathrm{e}}_{\{g=1,2,3\}}=\{\tilde{e}, \tilde{\mu}, \tilde{\tau}\}\right)$ we follow the discussion in Sect. 4 (option $\mathcal{O}_{2}$ ) of Refs. [51,52] and renormalize them on-shell. They then have to be computed from a mass matrix with shifted $M_{\tilde{L}}^{2}, M_{\tilde{Q}}^{2}, M_{\tilde{E}}^{2}$ and $M_{\tilde{D}}^{2}$; see below.

We apply the " $m_{b}, A_{b} \overline{\mathrm{DR}}$ " scheme of Refs. [51-53]. The scheme affecting sfermions $\tilde{\mathrm{e}}_{g}, \tilde{\mathrm{d}}_{g}$ is chosen with the variable \$SfScheme $[t, g]$ :

\$SfScheme $[2, g]=\mathrm{DR}[2]$

mixed scheme with $m_{\tilde{\mathrm{e}}_{2 g}} \mathrm{OS}, A_{\mathrm{e}_{g}} \overline{\mathrm{DR}}$,

\$SfScheme $[4, g]=\mathrm{DR}[2]$

mixed scheme with $m_{\tilde{\mathrm{d}}_{2 g}} \mathrm{OS}, A_{\mathrm{d}_{g}} \overline{\mathrm{DR}}$.

In the following, the sfermion index $s$ runs over both values 1, 2. All sfermions are on-shell,

$\operatorname{dMSf.sq} 1[1,1,1, g] \equiv \delta m_{\tilde{v}_{1 g}}^{2}=\widetilde{\operatorname{Re}}\left(\Sigma_{\tilde{v}_{g}}\left(m_{\tilde{v}_{1 g}}^{2}\right)\right)_{11}$,
$\operatorname{dMSfsq1}[s, s, 2, g] \equiv \delta m_{\tilde{\mathrm{e}}_{s g}}^{2}=\widetilde{\operatorname{Re}}\left(\Sigma_{\tilde{\mathrm{e}}_{g}}\left(m_{\tilde{\mathrm{e}}_{s g}}^{2}\right)\right)_{s s}$,

$\operatorname{dMSf.sq1}[s, s, 3, g] \equiv \delta m_{\tilde{\mathrm{u}}_{s g}}^{2}=\widetilde{\operatorname{Re}}\left(\Sigma_{\tilde{\mathrm{u}}_{g}}\left(m_{\tilde{\mathrm{u}}_{s g}}^{2}\right)\right)_{s s}$, 
$\operatorname{dMSfsq1}[s, s, 4, g] \equiv \delta m_{\tilde{\mathrm{d}}_{s g}}^{2}=\widetilde{\operatorname{Re}}\left(\Sigma_{\tilde{\mathrm{d}}_{g}}\left(m_{\tilde{\mathrm{d}}_{s g}}^{2}\right)\right)_{s s}$.

The up-type off-diagonal mass-matrix entries receive counterterms $[51,52,58,59]$

$$
\begin{aligned}
& \operatorname{dMSf.sq1}[1,2,3, g] \equiv \delta Y_{\mathrm{u}_{g}} \\
& \quad=\frac{1}{2} \widetilde{\operatorname{Re}}\left(\Sigma_{\tilde{\mathrm{u}}_{g}}\left(m_{\tilde{\mathrm{u}}_{1 g}}^{2}\right)+\Sigma_{\tilde{\mathrm{u}}_{g}}\left(m_{\tilde{\mathrm{u}}_{2 g}}^{2}\right)\right)_{12}, \\
& \operatorname{dMSf} \operatorname{sq} 1[2,1,3, g] \equiv \delta Y_{\mathrm{u}_{g}}^{*} \\
& =\frac{1}{2} \widetilde{\operatorname{Re}}\left(\Sigma_{\tilde{\mathrm{u}}_{g}}\left(m_{\tilde{\mathrm{u}}_{1 g}}^{2}\right)+\Sigma_{\tilde{\mathrm{u}}_{g}}\left(m_{\tilde{\mathrm{u}}_{2 g}}^{2}\right)\right)_{21} .
\end{aligned}
$$

For clarity of notation we furthermore define the auxiliary constants

$$
\begin{aligned}
\operatorname{dMsq12Sf1}[2, g] \equiv & \delta M_{\tilde{\mathrm{e}}_{g}, 12}^{2}=m_{\mathrm{e}_{g}}\left(\delta A_{\mathrm{e}_{g}}^{*}-\mu \delta t_{\beta}-t_{\beta} \delta \mu\right) \\
& +\left(A_{\mathrm{e}_{g}}^{*}-\mu t_{\beta}\right) \delta m_{\mathrm{e}_{g}}, \\
\text { aMsq12Sf1 }[4, g] \equiv & \delta M_{\tilde{\mathrm{d}}_{g}, 12}^{2}=m_{\mathrm{d}_{g}}\left(\delta A_{\mathrm{d}_{g}}^{*}-\mu \delta t_{\beta}-t_{\beta} \delta \mu\right) \\
& +\left(A_{\mathrm{d}_{g}}^{*}-\mu t_{\beta}\right) \delta m_{\mathrm{d}_{g}} .
\end{aligned}
$$

The electron/down-type off-diagonal mass counterterms are related by

aMSfsq1 $[1,2,2, g] \equiv \delta Y_{\mathrm{e}_{g}}$

$$
\begin{aligned}
= & \frac{1}{\left|U_{11}^{\tilde{\mathrm{e}}_{g}}\right|^{2}-\left|U_{12}^{\tilde{\mathrm{e}}_{g}}\right|^{2}}\left\{U_{11}^{\tilde{\mathrm{e}}_{g}} U_{21}^{\tilde{\mathrm{e}}_{g} *}\left(\delta m_{\tilde{\mathrm{e}}_{1 g}}^{2}-\delta m_{\tilde{\mathrm{e}}_{2 g}}^{2}\right)\right. \\
& \left.+U_{11}^{\tilde{\mathrm{e}}_{g}} U_{22}^{\tilde{\mathrm{e}}_{g} *} \delta M_{\tilde{\mathrm{e}}_{g}, 12}^{2}-U_{12}^{\tilde{\mathrm{e}}_{g}} U_{21}^{\tilde{\mathrm{e}}_{g} *} \delta M_{\tilde{\mathrm{e}}_{g}, 12}^{2 *}\right\},
\end{aligned}
$$

aMSfsq1 $[2,1,2, g]=\delta Y_{\mathrm{e}_{g}}^{*}$,

dMSfsq1 $[1,2,4, g] \equiv \delta Y_{\mathrm{d}_{g}}$

$$
\begin{aligned}
= & \frac{1}{\left|U_{11}^{\tilde{\mathrm{d}}_{g}}\right|^{2}-\left|U_{12}^{\tilde{\mathrm{d}}_{g}}\right|^{2}}\left\{U_{11}^{\tilde{\mathrm{d}}_{g}} U_{21}^{\tilde{\mathrm{d}}_{g} *}\left(\delta m_{\tilde{\mathrm{d}}_{1 g}}^{2}-\delta m_{\tilde{\mathrm{d}}_{2 g}}^{2}\right)\right. \\
& \left.+U_{11}^{\tilde{\mathrm{d}}_{g}} U_{22}^{\tilde{\mathrm{d}}_{g} *} \delta M_{\tilde{\mathrm{d}}_{g}, 12}^{2}-U_{12}^{\tilde{\mathrm{d}}_{g}} U_{21}^{\tilde{\mathrm{d}}_{g} *} \delta M_{\tilde{\mathrm{d}}_{g}, 12}^{2 *}\right\},
\end{aligned}
$$

aMSfsq1 $[2,1,4, g]=\delta Y_{\mathrm{d}_{g}}^{*}$.

The trilinear couplings $A_{f_{t g}} \equiv\left(\mathbf{A}_{f_{t}}\right)_{g g}$ are renormalized by

$$
\begin{aligned}
& \operatorname{dAf} 1[2, g, g] \equiv \delta A_{\mathrm{e}_{g}}=\left\{\frac { 1 } { m _ { \mathrm { e } _ { g } } } \left[U_{11}^{\tilde{\mathrm{e}}_{g}} U_{12}^{\tilde{\mathrm{e}}_{g} *}\left(\delta m_{\tilde{\mathrm{e}}_{1 g}}^{2}-\delta m_{\tilde{\mathrm{e}}_{2 g}}^{2}\right)\right.\right. \\
& \left.+U_{11}^{\tilde{\mathrm{e}}_{g}} U_{22}^{\tilde{\mathrm{e}}_{g} *} \delta Y_{\mathrm{e}_{g}}^{*}+U_{12}^{\tilde{\mathrm{e}}_{g} *} U_{21}^{\tilde{\mathrm{e}}_{g}} \delta Y_{\mathrm{e}_{g}}-\left(A_{\mathrm{e}_{g}}-\mu^{*} t_{\beta}\right) \delta m_{\mathrm{e}_{g}}\right] \\
& \left.+\delta \mu^{*} t_{\beta}+\mu^{*} \delta t_{\beta}\right\}_{\text {div }},
\end{aligned}
$$

$$
\begin{aligned}
& \operatorname{dAf} 1[3, g, g] \equiv \delta A_{\mathrm{u}_{g}}=\frac{1}{m_{\mathrm{u}_{g}}}\left[U_{11}^{\tilde{\mathrm{u}}_{g}} U_{12}^{\tilde{\mathrm{u}}_{g} *}\left(\delta m_{\tilde{\mathrm{u}}_{1 g}}^{2}-\delta m_{\tilde{\mathrm{u}}_{2 g}}^{2}\right)\right. \\
& \left.\quad+U_{11}^{\tilde{\mathrm{u}}_{g}} U_{22}^{\tilde{\mathrm{u}}_{g} *} \delta Y_{\mathrm{u}_{g}}^{*}+U_{12}^{\tilde{\mathrm{u}}_{g} *} U_{21}^{\tilde{\mathrm{u}}_{g}} \delta Y_{\mathrm{u}_{g}}-\left(A_{\mathrm{u}_{g}}-\mu^{*} / t_{\beta}\right) \delta m_{\mathrm{u}_{g}}\right] \\
& \quad+\delta \mu^{*} / t_{\beta}-\mu^{*} \delta t_{\beta} / t_{\beta}^{2},
\end{aligned}
$$

$\mathrm{dAf} 1[4, g, g] \equiv \delta A_{\mathrm{d}_{g}}=\left\{\frac{1}{m_{\mathrm{d}_{g}}}\left[U_{11}^{\tilde{\mathrm{d}}_{g}} U_{12}^{\tilde{\mathrm{d}}_{g} *}\left(\delta m_{\tilde{\mathrm{d}}_{1 g}}^{2}-\delta m_{\tilde{\mathrm{d}}_{2 g}}^{2}\right)\right.\right.$

$$
\left.+U_{11}^{\tilde{\mathrm{d}}_{g}} U_{22}^{\tilde{\mathrm{d}}_{g} *} \delta Y_{\mathrm{d}_{g}}^{*}+U_{12}^{\tilde{\mathrm{d}}_{g} *} U_{21}^{\tilde{\mathrm{d}}_{g}} \delta Y_{\mathrm{d}_{g}}-\left(A_{\mathrm{d}_{g}}-\mu^{*} t_{\beta}\right) \delta m_{\mathrm{d}_{g}}\right]
$$$$
\left.+\delta \mu^{*} t_{\beta}+\mu^{*} \delta t_{\beta}\right\}_{\mathrm{div}} \text {, }
$$

where the subscripted "div" means to take the divergent part, to effect $\overline{\mathrm{DR}}$ renormalization of $A_{\mathrm{e}_{g}}$ and $A_{\mathrm{d}_{g}}$ [51,52].

As now all the sfermion masses are renormalized as onshell an explicit restoration of the $S U$ (2) relation is needed. This is performed in requiring that the left-handed (bare) soft SUSY-breaking mass parameter $M_{\tilde{Q}}\left(M_{\tilde{L}}\right)$ is the same in the $\tilde{\mathrm{d}}_{g}\left(\tilde{\mathrm{e}}_{g}\right)$ as in the $\tilde{\mathrm{u}}_{g}\left(\tilde{\mathrm{v}}_{g}\right)$ sfermion sector at the one-loop level (see also Refs. [60-63]),

$M_{\tilde{L}}^{2}\left(\tilde{\mathrm{e}}_{g}\right)=M_{\tilde{L}}^{2}\left(\tilde{v}_{g}\right)+\delta M_{\tilde{L}}^{2}\left(\tilde{v}_{g}\right)-\delta M_{\tilde{L}}^{2}\left(\tilde{\mathrm{e}}_{g}\right)$,
$M_{\tilde{Q}}^{2}\left(\tilde{\mathrm{d}}_{g}\right)=M_{\tilde{Q}}^{2}\left(\tilde{\mathrm{u}}_{g}\right)+\delta M_{\tilde{Q}}^{2}\left(\tilde{\mathrm{u}}_{g}\right)-\delta M_{\tilde{Q}}^{2}\left(\tilde{\mathrm{d}}_{g}\right)$

with

$$
\begin{aligned}
\delta M_{\tilde{L}, \tilde{Q}}^{2}\left(\tilde{f}_{g}\right)= & \left|U_{11}^{\tilde{f}_{g}}\right|^{2} \delta m_{\tilde{f}_{1 g}}^{2}+\left|U_{12}^{\tilde{f}_{g}}\right|^{2} \delta m_{\tilde{f}_{2 g}}^{2}-U_{22}^{\tilde{f}_{g}} U_{12}^{\tilde{f}_{g} *} \delta Y_{f_{g}} \\
& -U_{12}^{\tilde{f}_{g}} U_{22}^{\tilde{f}_{g} *} \delta Y_{f_{g}}^{*}-2 m_{f_{g}} \delta m_{f_{g}} \\
& +M_{Z}^{2} c_{2 \beta} Q_{f_{g}}, \delta s_{\mathrm{W}}^{2}-\left(I_{3}^{f_{g}}-Q_{f_{g}} s_{\mathrm{W}}^{2}\right) \\
& \times\left(c_{2 \beta} \delta M_{Z}^{2}+M_{Z}^{2} \delta c_{2 \beta}\right) .
\end{aligned}
$$

Now $M_{\tilde{L}}^{2}\left(\tilde{\mathrm{e}}_{g}\right)$ and $M_{\tilde{Q}}^{2}\left(\tilde{\mathrm{d}}_{g}\right)$ are used in the scalar mass matrix instead of the parameters $M_{\tilde{L}, \tilde{Q}}^{2}$ in Eq. (3) when calculating the values of $m_{\tilde{\mathrm{e}}_{s g}}$ and $m_{\tilde{\mathrm{d}}_{s g}}$. However, with this procedure, also $m_{\tilde{\mathrm{e}}_{2 g}}$ and $m_{\tilde{\mathrm{d}}_{2 g}}$ are shifted, which contradicts our choice of independent parameters. To keep this choice, also the righthanded soft SUSY-breaking mass parameters $M_{\tilde{E}, \tilde{D}}^{2}$ receive a shift: ${ }^{4}$

$$
\begin{aligned}
M_{\tilde{E}}^{2}\left(\tilde{\mathrm{e}}_{g}\right)= & \frac{m_{\mathrm{e}_{g}}^{2}\left|X_{\mathrm{e}_{g}}\right|^{2}}{M_{\tilde{L}}^{2}\left(\tilde{\mathrm{e}}_{g}\right)+m_{\mathrm{e}_{g}}^{2}+c_{2 \beta} M_{Z}^{2}\left(I_{3}^{\mathrm{e}}-Q_{\mathrm{e}} s_{\mathrm{W}}^{2}\right)-m_{\tilde{\mathrm{e}}_{2 g}}^{2}} \\
& -m_{\mathrm{e}_{g}}^{2}-c_{2 \beta} M_{Z}^{2} Q_{\mathrm{e}} s_{\mathrm{W}}^{2}+m_{\tilde{\mathrm{e}}_{2 g}}^{2},
\end{aligned}
$$

\footnotetext{
${ }^{4}$ If the mass of the $\tilde{\mathrm{d}}_{1 g}\left(\tilde{\mathrm{e}}_{1 g}\right)$ sfermion is chosen as independent mass as $\tilde{\mathrm{d}}_{2 g} \approx \tilde{\mathrm{d}}_{L g}\left(\tilde{\mathrm{e}}_{2 g} \approx \tilde{\mathrm{e}}_{L g}\right)$ then the shift of $M_{\tilde{D}}^{2}\left(M_{\tilde{E}}^{2}\right)$ has to be performed with respect to $m_{\tilde{\mathrm{d}}_{1 g}}\left(m_{\tilde{\mathrm{e}}_{1 g}}\right)$.
} 


$$
\begin{aligned}
M_{\tilde{D}}^{2}\left(\tilde{\mathrm{d}}_{g}\right)= & \frac{m_{\mathrm{d}_{g}}^{2}\left|X_{\mathrm{d}_{g}}\right|^{2}}{M_{\tilde{Q}}^{2}\left(\tilde{\mathrm{d}}_{g}\right)+m_{\mathrm{d}_{g}}^{2}+c_{2 \beta} M_{Z}^{2}\left(I_{3}^{\mathrm{d}}-Q_{\mathrm{d}} s_{\mathrm{w}}^{2}\right)-m_{\tilde{\mathrm{d}}_{2 g}}^{2}} \\
& -m_{\mathrm{d}_{g}}^{2}-c_{2 \beta} M_{Z}^{2} Q_{\mathrm{d}} s_{\mathrm{w}}^{2}+m_{\tilde{\mathrm{d}}_{2 g}}^{2} .
\end{aligned}
$$

Taking into account this shift in $M_{\tilde{E}, \tilde{D}}^{2}$, up to one-loop order, ${ }^{5}$ the new resulting mass parameters $m_{\tilde{\mathrm{e}}_{1 g}}$ and $m_{\tilde{\mathrm{d}}_{1 g}}$ are the same as the on-shell masses

$$
\begin{aligned}
& \left(m_{\tilde{\mathrm{e}}_{1 g}}^{\mathrm{OS}}\right)^{2}=m_{\tilde{\mathrm{e}}_{1 g}}^{2}+\delta m_{\tilde{\mathrm{e}}_{1 g}}^{2}-\widetilde{\operatorname{Re}}\left(\Sigma_{\tilde{\mathrm{e}}_{g}}\left(m_{\tilde{\tilde{\mathrm{e}}}_{1 g}}^{2}\right)\right)_{11}, \\
& \left(m_{\tilde{\mathrm{d}}_{1 g}}^{\mathrm{OS}}\right)^{2}=m_{\tilde{\mathrm{d}}_{1 g}}^{2}+\delta m_{\tilde{\mathrm{d}}_{1 g}}^{2}-\widetilde{\operatorname{Re}}\left(\Sigma_{\tilde{\mathrm{d}}_{g}}\left(m_{\tilde{\mathrm{d}}_{1 g}}^{2}\right)\right)_{11} .
\end{aligned}
$$

The input parameters in the $b / \tilde{b}$ sector have to correspond to the chosen renormalization. We start by defining the bottom mass, where the experimental input is the SM $\overline{M S}$ mass [66],

$m_{b}^{\overline{\mathrm{MS}}}\left(m_{b}\right)=4.18 \mathrm{GeV}$.

To convert to the $\overline{\mathrm{DR}}$ mass the following procedure is chosen. The value of $m_{b}^{\overline{\mathrm{MS}}}\left(\mu_{R}\right)$ (at the renormalization scale $\mu_{R}$ ) is calculated from $m_{b}^{\overline{\mathrm{MS}}}\left(m_{b}\right)$ at the three-loop level

$m_{b}^{\overline{\mathrm{MS}}}\left(\mu_{R}\right)=m_{b}^{\overline{\mathrm{MS}}}\left(m_{b}\right) \frac{c\left(\alpha_{s}^{\overline{\mathrm{MS}},\left(n_{f}\right)}\left(\mu_{R}\right) / \pi\right)}{c\left(\alpha_{s}^{\overline{\mathrm{MS}},\left(n_{f}\right)}\left(m_{b}\right) / \pi\right)}$

via the function $c(x)$ following the prescription given in Refs. $[67,68] . n_{f}$ denotes the number of active flavors. The "onshell" mass is connected to the $\overline{\mathrm{MS}}$ mass via

$$
\begin{aligned}
& m_{b}^{\mathrm{os}}=m_{b}^{\overline{\mathrm{MS}}}\left(\mu_{R}\right) \\
& \times\left[1+\frac{\alpha_{s}^{\overline{\mathrm{MS}},\left(n_{f}\right)}\left(\mu_{R}\right)}{\pi}\left(\frac{4}{3}+2 \ln \frac{\mu_{R}}{m_{b}^{\overline{\mathrm{MS}}}\left(\mu_{R}\right)}\right)+\cdots\right],
\end{aligned}
$$

where the ellipsis denote the two- and three-loop contributions, which can also be found in Refs. [67,68]. The $\overline{\mathrm{DR}}$ bottom quark mass at the scale $\mu_{R}$ is calculated iteratively from $[59,65,69]$

$m_{b}^{\overline{\mathrm{DR}}}=m_{b}^{\mathrm{os}}+\frac{\delta m_{b}^{\mathrm{OS}}-\delta m_{b}^{\overline{\mathrm{DR}}}}{\left|1+\Delta_{b}\right|}$

with an accuracy of $\left|1-\left(m_{b}^{\overline{\mathrm{DR}}}\right)^{(n)} /\left(m_{b}^{\overline{\mathrm{DR}}}\right)^{(n-1)}\right|<10^{-5}$ reached in the $n$th step of the iteration.

\footnotetext{
${ }^{5}$ In the case of a pure OS scheme (see e.g. [64,65] for the rMSSM) the shifts Eqs. (12) and (14) result in mass parameters $m_{\tilde{\mathrm{d}}_{1 g}}$ and $m_{\tilde{\mathrm{e}}_{1 g}}$, which are exactly the same as in Eq. (15). This constitutes an important consistency check of these two different methods.
}

The quantity $\Delta_{b}[25,69-72]$ (see also Ref. [73]) resums the $\mathcal{O}\left(\left(\alpha_{s} t_{\beta}\right)^{n}\right)$ and $\mathcal{O}\left(\left(\alpha_{t} t_{\beta}\right)^{n}\right)$ terms and is given by

$$
\begin{aligned}
\Delta_{b}= & -\frac{\mu^{*} t_{\beta}}{\pi}\left[\frac{2}{3} \alpha_{s}\left(m_{t}\right) M_{3}^{*} I\left(m_{\tilde{b}_{1}}^{2}, m_{\tilde{b}_{2}}^{2}, m_{\tilde{g}}^{2}\right)\right. \\
& \left.+\frac{1}{4} \alpha_{t}\left(m_{t}\right) A_{t}^{*} I\left(m_{\tilde{t}_{1}}^{2}, m_{\tilde{t}_{2}}^{2},|\mu|^{2}\right)\right]
\end{aligned}
$$

with

$$
\begin{aligned}
I(a, b, c) & =C_{0}(0,0,0, a, b, c) \\
& =-\frac{a b \ln (b / a)+a c \ln (a / c)+b c \ln (c / b)}{(c-a)(c-b)(b-a)} .
\end{aligned}
$$

Here $\alpha_{t}$ is defined in terms of the top Yukawa coupling $y_{t}\left(m_{t}\right)=\sqrt{2} m_{t}\left(m_{t}\right) / v$ as $\alpha_{t}\left(m_{t}\right)=y_{t}^{2}\left(m_{t}\right) /(4 \pi)$ with $v=1 / \sqrt{\sqrt{2} G_{F}}=246.218 \mathrm{GeV}$, and $m_{t}\left(m_{t}\right) \approx m_{t} /(1-$ $\left.\frac{1}{2 \pi} \alpha_{t}\left(m_{t}\right)+\frac{4}{3 \pi} \alpha_{s}\left(m_{t}\right)\right)$. Setting in the evaluation of $\Delta_{b}$ the scale to $m_{t}$ was shown to yield in general a more stable result $[22,23]$ as long as two-loop corrections to $\Delta_{b}$ are not included. ${ }^{6} M_{3}$ is the soft SUSY-breaking parameter for the gluinos. We have neglected any CKM mixing of the quarks.

The $Z$ factors of the squark fields are derived in the OS scheme. They can be found in Ref. [57].

\subsection{The strong coupling constant}

The decoupling of the heavy particles and the running is taken into account in the definition of $\alpha_{s}$ : the starting point is [66]

$\alpha_{s}^{\overline{\mathrm{MS}},(5)}\left(M_{Z}\right)=0.1184$,

where the running of $\alpha_{s}^{\overline{\mathrm{MS}},\left(n_{f}\right)}\left(\mu_{R}\right)$ can be found in Ref. [66]. $\mu_{R}$ denotes the renormalization scale, which is typically of the order of the energy scale of the considered process.

From the $\overline{\mathrm{MS}}$ value the $\overline{\mathrm{DR}}$ value is obtained at the twoloop level via the phenomenological one-step formula [74, 75]

$$
\begin{aligned}
& \alpha_{s}^{\overline{\mathrm{DR}},\left(n_{f}\right)}\left(\mu_{R}\right)=\alpha_{s}^{\overline{\mathrm{MS}},\left(n_{f}\right)}\left(\mu_{R}\right)\left\{1+\frac{\alpha_{s}^{\overline{\mathrm{MS}},\left(n_{f}\right)}\left(\mu_{R}\right)}{\pi}\right. \\
& \quad \times\left(\frac{1}{4}-\zeta_{1}^{\left(n_{f}\right)}\right)+\left(\frac{\alpha_{s}^{\overline{\mathrm{MS}},\left(n_{f}\right)}\left(\mu_{R}\right)}{\pi}\right)^{2} \\
& \left.\quad \times\left[\frac{11}{8}-\frac{n_{f}}{12}-\frac{1}{2} \zeta_{1}^{\left(n_{f}\right)}+2\left(\zeta_{1}^{\left(n_{f}\right)}\right)^{2}-\zeta_{2}^{\left(n_{f}\right)}\right]\right\},
\end{aligned}
$$

\footnotetext{
${ }^{6}$ It should be noted that in Refs. [22,23] a different scale has been advocated due to the emphasis on the two-loop contributions presented in this paper. The plots, however, show that $m_{t}$ is a good scale choice if only one-loop corrections are included.
} 

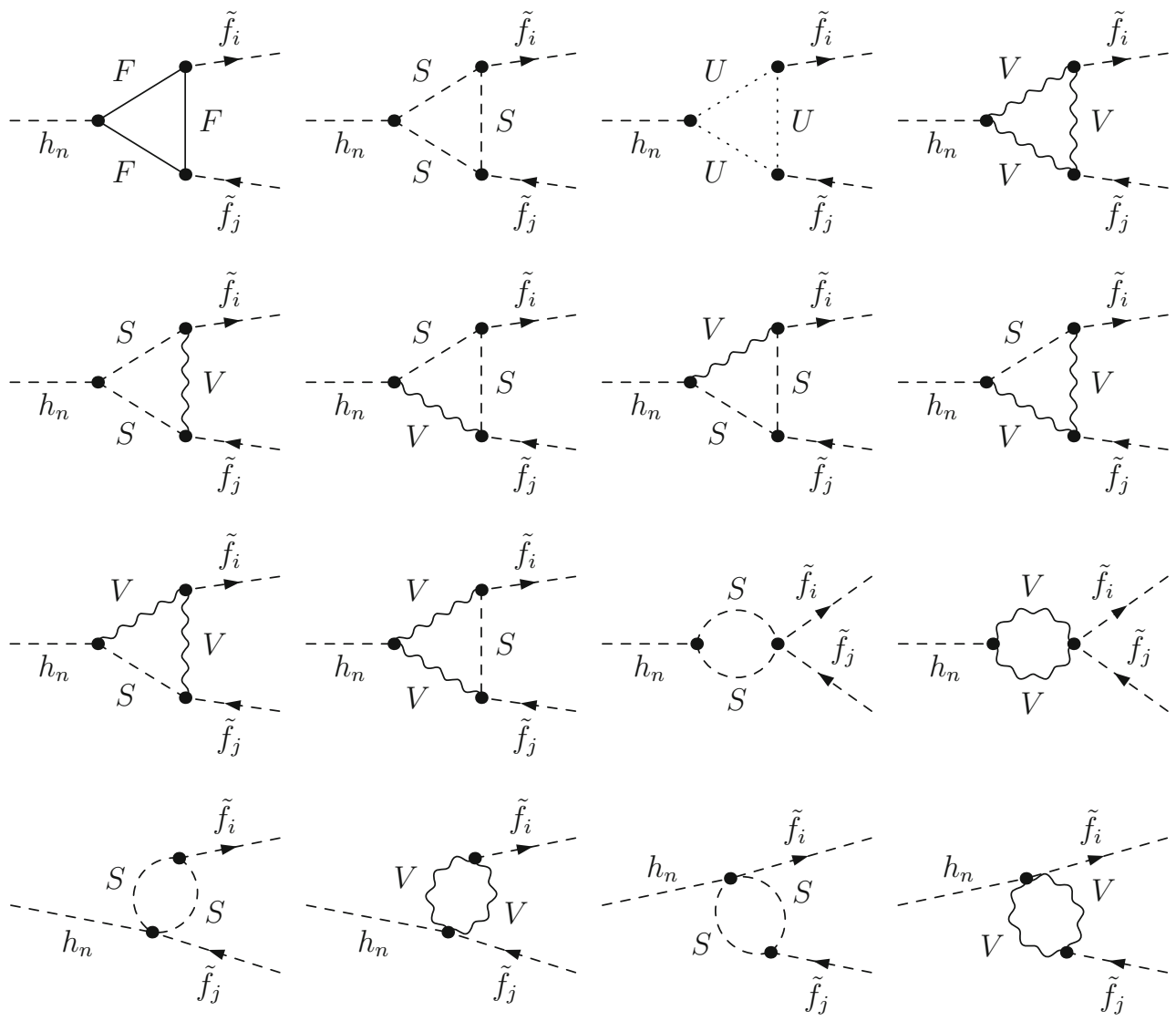

Fig. 1 Generic Feynman diagrams for the decay $h_{n} \rightarrow \tilde{f}_{i} \tilde{f}_{j}(n=$ $2,3 ; i, j=1,2)$. $F$ can be a SM fermion, chargino, neutralino, or gluino; $S$ can be a sfermion or a Higgs/Goldstone boson; $U$ denotes the

ghosts; $V$ can be a $\gamma, Z, W^{ \pm}$, or $g$. Not shown are the diagrams with a $h_{n}-Z$ or $h_{n}-G$ transition contribution on the external Higgs-boson leg

where (for $n_{f}=6$ )

$\zeta_{1}^{(6)}=-\ln \frac{\mu_{R}^{2}}{\tilde{M}^{2}}, \quad \zeta_{2}^{(6)}=-\frac{65}{32}-\frac{5}{2} \ln \frac{\mu_{R}^{2}}{\tilde{M}^{2}}+\left(\ln \frac{\mu_{R}^{2}}{\tilde{M}^{2}}\right)^{2}$

with $\tilde{M}^{2}$ being defined as the geometric average of all squark masses multiplied with the gluino mass, ${ }^{7} \tilde{M}^{2}=$ $m_{\tilde{g}} \prod_{\tilde{q}}\left(m_{\tilde{q}_{1}} m_{\tilde{q}_{2}}\right)^{\frac{1}{12}}$. The log terms originates from the decoupling of the SQCD particles from the running of $\alpha_{s}$ at lower scales $\mu_{R} \leq \mu_{\mathrm{dec}}$. Here $\mu_{\mathrm{dec}}$ denotes the decoupling scale for heavy particles, i.e. the scale where intermediate effective theories are introduced by integrating out the heavy degrees of freedom. For simplification we have chosen the energy scale of the considered processes (as a typical SUSY scale) also as decoupling scale.

$7 \tilde{M}$ is chosen such that $\ln \frac{m_{\tilde{\tilde{g}}}^{2}}{\tilde{\tilde{M}}^{2}}+\frac{1}{6} \sum_{\tilde{q}} \ln \frac{m_{\tilde{q}_{1}} m_{\tilde{q}_{2}}}{\tilde{M}^{2}}=0$, which means that the corresponding diagrams vanish at zero momentum transfer. Under this condition $\alpha_{S}\left(\mu_{R}\right)$ is well defined.

\section{Calculation of loop diagrams}

In this section we give some details about the calculation of the higher-order corrections to the partial decay widths of Higgs bosons. Sample diagrams are shown in Figs. 1 and 2. Not shown are the diagrams for real (hard and soft) photon and gluon radiation. They are obtained from the corresponding tree-level diagrams by attaching a photon (gluon) to the electrically (color) charged particles. The internal generically depicted particles in Figs. 1 and 2 are labeled as follows: $F$ can be a SM fermion $f$, chargino $\tilde{\chi}_{j}^{ \pm}$, neutralino $\tilde{\chi}_{k}^{0}$, or gluino $\tilde{g} ; S$ can be a sfermion $\tilde{f}_{i}$ or a Higgs (Goldstone) boson $h_{n}(G) ; U$ denotes the ghosts $u_{V} ; V$ can be a photon $\gamma$, gluon $g$, or a massive SM gauge boson, $Z$ or $W^{ \pm}$. For internally appearing Higgs bosons no higher-order corrections to their masses or couplings are taken into account; these corrections would correspond to effects beyond one-loop order. ${ }^{8}$ For external Higgs bosons, as described in Sect. 2.1,

\footnotetext{
8 We found that using loop corrected Higgs-boson masses in the loops leads to a UV divergent result.
} 

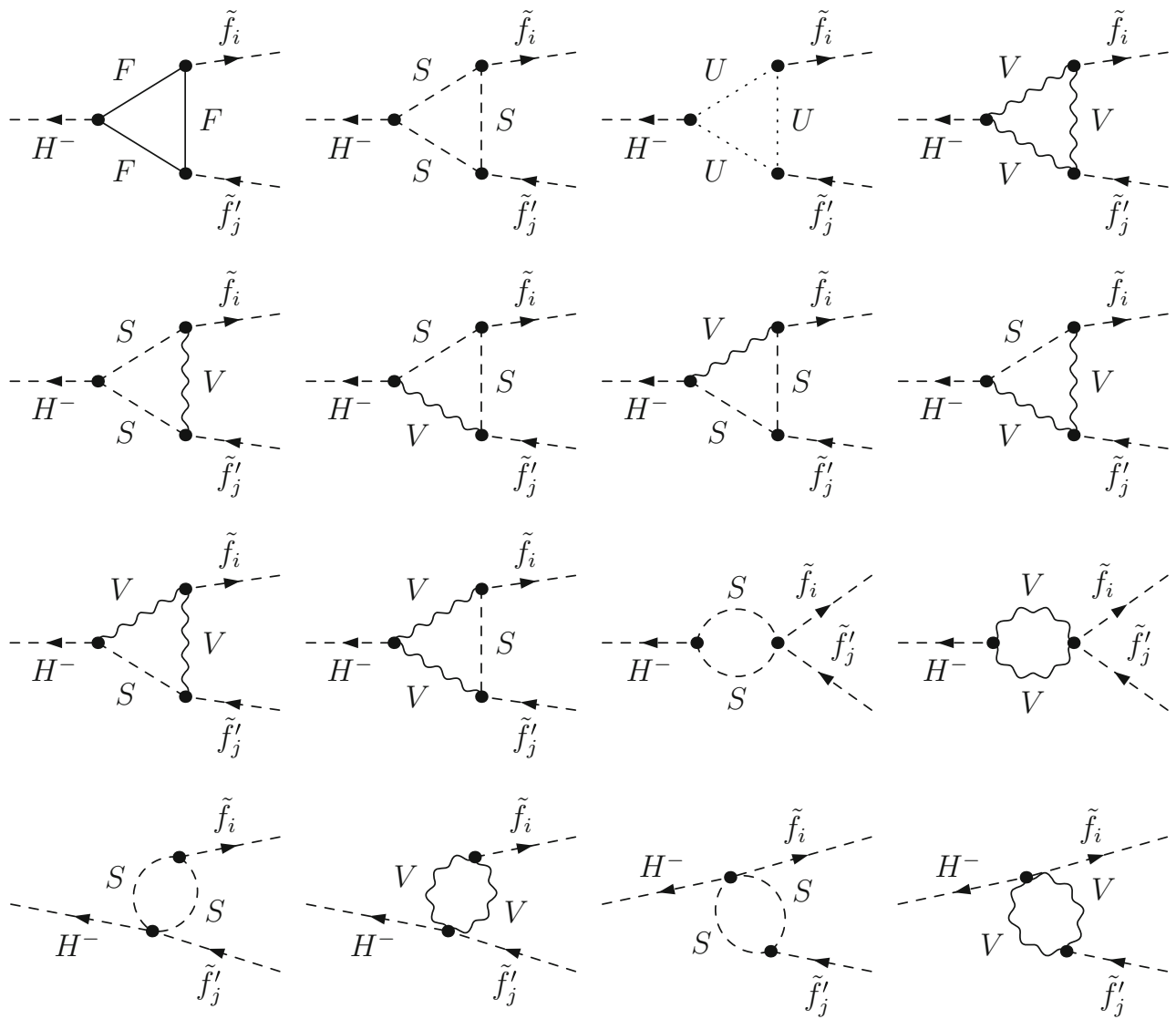

Fig. 2 Generic Feynman diagrams for the decay $H^{+} \rightarrow \tilde{f}_{i} \tilde{f}_{j}^{\prime}(i, j=$ $1,2)$. (It should be noted that all arrows are inverted in case of a $\mathrm{H}^{-}$ decay.) $F$ can be a SM fermion, chargino, neutralino, or gluino; $S$ can

be a sfermion or a Higgs/Goldstone boson; $U$ denotes the ghosts; $V$ can be a $\gamma, Z, W^{ \pm}$, or $g$. Not shown are the diagrams with a $H^{ \pm}-W^{ \pm}$or $H^{ \pm}-G^{ \pm}$transition contribution on the external Higgs-boson leg

the appropriate $\hat{Z}$ factors are applied and on-shell masses (including higher-order corrections) are used [33], obtained with FeynHiggs [33-38].

Also not shown are the diagrams with a Higgs-gauge/ Goldstone boson self-energy contribution on the external Higgs-boson leg. They appear in the decay $h_{n} \rightarrow \tilde{f}_{i} \tilde{f}_{j}$, Fig. 1, with a $h_{n}-Z / G$ transition and in the decay $H^{ \pm} \rightarrow$ $\tilde{f}_{i} \tilde{f}_{j}^{\prime}$, Fig. 2 , with a $H^{ \pm}-W^{ \pm} / G^{ \pm}$transition. ${ }^{9}$

Furthermore, in general, in Figs. 1 and 2 we have omitted diagrams with self-energy type corrections of external (onshell) particles. While the contributions from the real parts of the loop functions are taken into account via the renormalization constants defined by on-shell renormalization conditions, the contributions coming from the imaginary part of the loop functions can result in an additional (real) correction if multiplied by complex parameters (such as $A_{f}$ ). In the analytical and numerical evaluation, these diagrams have

${ }_{9}$ From a technical point of view, the $H^{ \pm}-W^{ \pm} / G^{ \pm}$transitions have been absorbed into the respective counterterms, while the $h_{n}-Z / G$ transitions have been calculated explicitly.

been taken into account via the prescription described in Ref. [57].

Within our one-loop calculation we neglect finite width effects that can help to cure threshold singularities. Consequently, in the close vicinity of those thresholds our calculation does not give a reliable result. Switching to a complex mass scheme [76] would be another possibility to cure this problem, but its application is beyond the scope of our paper.

The diagrams and corresponding amplitudes have been obtained with FeynArts [77-79]. The model file, including the MSSM counterterms, is largely based on Ref. [57]; however, adjusted to match exactly the renormalization prescription described in Sect. 2. The further evaluation has been performed with FormCalc and LoopTools [80].

\subsection{Ultraviolet divergences}

As regularization scheme for the UV divergences we have used constrained differential renormalization [81], which has been shown to be equivalent to dimensional reduction $[82,83]$ at the one-loop level [80]. Thus the employed regulariza- 
tion scheme preserves SUSY $[84,85]$ and guarantees that the SUSY relations are kept intact, e.g. that the gauge couplings of the SM vertices and the Yukawa couplings of the corresponding SUSY vertices also coincide to one-loop order in the SUSY limit. Therefore no additional shifts, which might occur when using a different regularization scheme, arise. All UV divergences cancel in the final result.

\subsection{Infrared divergences}

The IR divergences from diagrams with an internal photon or gluon have to cancel with the ones from the corresponding real soft radiation. In the case of QED we have included the soft photon contribution following the description given in Ref. [86]. In the case of QCD we have modified this prescription by replacing the product of electric charges by the appropriate combination of color charges (linear combination of $C_{A}$ and $C_{F}$ times $\alpha_{S}$ ).

The IR divergences arising from the diagrams involving a $\gamma$ (or a $g$ ) are regularized by introducing a photon (or gluon) mass parameter, $\lambda$. While for the QED part this procedure always works, in the QCD part due to its non-Abelian character this method can fail. However, since no triple or quartic gluon vertices appear, $\lambda$ can indeed be used as a regulator. Using shifted (i.e. on-shell) sfermion masses inside tree- and loop-level calculations guarantees that all IR divergences, i.e. all divergences in the limit $\lambda \rightarrow 0$, cancel once virtual and real diagrams for one decay channel are added.

\subsection{Tree-level formulas}

For completeness we show here also the formulas that have been used to calculate the tree-level decay widths:

$$
\begin{aligned}
\Gamma^{\text {tree }}\left(h_{n} \rightarrow \tilde{f}_{i} \tilde{f}_{j}\right)= & \frac{\left|C\left(h_{n}, \tilde{f}_{i}, \tilde{f}_{j}\right)\right|^{2} \lambda^{1 / 2}\left(m_{h_{n}}^{2}, m_{\tilde{f}_{i}}^{2}, m_{\tilde{f}_{j}}^{2}\right)}{16 \pi m_{h_{n}}^{3}} \\
\Gamma^{\text {tree }}\left(H^{ \pm} \rightarrow \tilde{f}_{i} \tilde{f}_{j}^{\prime}\right)= & \times \frac{\left|C\left(H^{ \pm}, \tilde{f}_{i}, \tilde{f}_{j}^{\prime}\right)\right|^{2} \lambda^{1 / 2}\left(M_{H^{ \pm}}^{2}, m_{\tilde{f}_{i}}^{2}, m_{\tilde{f}_{j}^{\prime}}^{2}\right)}{16 \pi M_{H^{ \pm}}^{3}} \\
& \times(i, j=1,2),
\end{aligned}
$$

where $\lambda(x, y, z)=(x-y-z)^{2}-4 y z$ and the couplings $C(a, b, c)$ can be found in the FeynArts model files [87].

\section{Numerical analysis}

In this section we present the comparisons with results from other groups and our numerical analysis of all heavy Higgsboson decay channels into the third generation sfermions in the cMSSM. In the various figures below we show the partial decay widths and their relative correction at the tree level ("tree") and at the one-loop level ("full"). In addition we show the SQCD corrections ("SQCD") for comparison with the full one-loop result.

\subsection{Comparisons}

We performed exhaustive comparisons with results from other groups for heavy Higgs-boson decays. Since loop corrections in the MSSM with complex parameters have been evaluated in this work for the first time, these comparisons were restricted to the MSSM with real parameters.

- We calculated the decays $\Phi \rightarrow \tilde{q}_{i} \tilde{q}_{j}$ at $\mathcal{O}\left(\alpha_{s}\right)$ ( $\Phi$ denotes any heavy MSSM Higgs boson) and found good agreement with Ref. [55], where only a small difference remains due to the slightly different renormalization schemes. We successfully reproduced their figures, except their Fig. $4\left(H^{+} \rightarrow \tilde{t}_{1} \tilde{b}_{1}\right)$, which differs substantially. Unfortunately, 17 years after publication, the source code of Ref. [55] is unavailable for a direct comparison [88]. On the other hand our results for $\mathrm{H}^{+} \rightarrow$ $\tilde{t}_{1} \tilde{b}_{1}$ are in good qualitative agreement with Ref. [46]; see below.

- A comparison with Ref. [56] at $\mathcal{O}\left(\alpha_{s}\right)$ was rather difficult. Reference [56] used running $\overline{\mathrm{MS}}$ input parameters and significant differences exist w.r.t. our treatment of the renormalization of the scalar quark sector. Nevertheless, using their input parameters as far as possible, we found qualitative agreement.

- We performed a detailed comparison with Ref. [44] for the decay $H^{+} \rightarrow \tilde{t}_{i} \tilde{b}_{j}$ at $\mathcal{O}\left(\alpha_{s}\right)$. They also differ in the renormalization of the scalar quark sector, leading to different loop corrections. Furthermore, they used tree/ $\overline{\mathrm{DR}} /$ pole squark masses in tree/loop/phase space. Despite these complications we found rather good qualitative agreement with their Fig. 2.

- A check with Refs. [45] and [46] at $\mathcal{O}\left(\alpha_{s}\right)$ gave good qualitative agreement, although an effective resummation of the trilinear Higgs-sbottom coupling was used in Ref. [46].

- Decays of the $\mathcal{C P}$-odd Higgs boson $A$ to scalar quarks (in the rMSSM) have been compared with Ref. [47]. Again, using their input parameters as far as possible, we found good (qualitative) agreement with their Figs. 4-7.

- A boson decays into sfermions in the rMSSM have been analyzed in Ref. [48]. As in the latter item we found good (qualitative) agreement, especially for the decay into scalar taus after using our new $\overline{\mathrm{DR}}$ type version for the slepton sector, as described above in Sect. 2.4.

- A numerical comparison with the program HFOLD Ref. [50] at the benchmark point SPS1a' (proposed in the SPA project [89]) can be found in Table 1. Only for this point sufficient details about the sfermion masses was 
Table 1 Comparison of the 1-loop corrected partial decay widths in $10^{-2} \mathrm{GeV}$
${ }^{a}$ It should be noted that in HFOLD an additional factor $1 / 2$ appears in the decay to sneutrinos. We omitted this (wrong) factor in the comparison

\begin{tabular}{llllll}
\hline Process & \multicolumn{2}{l}{ OS masses } & & \multicolumn{1}{c}{$\overline{\text { DR masses }}$} \\
\cline { 2 - 3 } \cline { 5 - 6 } & HFOLD & FeynTools & & HFOLD & FeynTools \\
\hline$H^{0} \rightarrow \tilde{\tau}_{1} \tilde{\tau}_{1}$ & 1.8426 & 2.0855 & 1.9866 & 2.2733 \\
$H^{0} \rightarrow \tilde{\tau}_{1} \tilde{\tau}_{2}$ & 1.9065 & 1.9001 & & 1.8187 & 1.8160 \\
$H^{0} \rightarrow \tilde{\tau}_{2} \tilde{\tau}_{2}$ & 0.3506 & 0.4399 & & - & - \\
$H^{0} \rightarrow \tilde{v}_{\tau} \tilde{\nu}_{\tau}{ }^{a}$ & 0.1070 & 0.1263 & & 0.0869 & 0.0997 \\
$A^{0} \rightarrow \tilde{\tau}_{1} \tilde{\tau}_{2}$ & 2.9686 & 3.1641 & & 2.8278 & 3.0212 \\
$H^{+} \rightarrow \tilde{v}_{\tau} \tilde{\tau}_{1}$ & 6.4323 & 7.0313 & & 6.5677 & 7.0049 \\
$H^{+} \rightarrow \tilde{v}_{\tau} \tilde{\tau}_{2}$ & 0.1180 & 0.1613 & & 0.0768 & 0.1022 \\
\hline
\end{tabular}

available for a numerical comparison. (Unfortunately, for this point only decays to scalar leptons are kinematically allowed.) In Table 1 we show the full one-loop results of HFOLD, using $\overline{\mathrm{DR}}$ masses for the internal and external particles, corresponding to the full $\overline{\mathrm{DR}}$ renormalization used in the code (where the renormalization scale was set to $1 \mathrm{TeV}$ [89]). Our results, labeled FeynTools, are evaluated using our renormalization scheme, but inserting the HFOLD $\overline{\mathrm{DR}}$ masses, and we find overall agreement better than $10 \%$ (in the tree-level results we find more than 10 digits agreement). ${ }^{10}$

HFOLD also offers to switch to (the recommended) OS masses for the external particles. In this case, including in our calculation the shifts to OS masses as described in Sect. 2.4, again with overall agreement between the two calculations roughly at the $10 \%$ level. The numerical difference between the two variants ( $\overline{\mathrm{DR}}$ and $\mathrm{OS})$ again is roughly at the $10 \%$ level. The fact that our results differ at the same level in the two schemes indicates that the numerical differences are mostly due to the different renormalization schemes used in our calculation and in HFOLD.

Finally, it should be noted that Refs. [44-48] subsequently had been recompiled in Ref. [49].

\subsection{Parameter settings}

The renormalization scale $\mu_{R}$ has been set to the mass of the decaying Higgs boson. The SM parameters are chosen as follows; see also [66]:

\footnotetext{
$\overline{10}$ It should be noted that we found results substantially closer to the HFOLD $\overline{D R}$ result using a "quasi" $\overline{\mathrm{DR}}$ scheme [where "quasi" means that our analytic integrals for the real radiation contain a finite piece and thus cannot easily be matched to pure $\overline{\mathrm{DR}}$ vertex renormalizations (of IR divergent diagrams)].
}

- Fermion masses (on-shell masses, if not indicated differently):

$$
\begin{aligned}
m_{e} & =0.510998928 \mathrm{MeV}, \quad m_{v_{e}}=0 \mathrm{MeV}, \\
m_{\mu} & =105.65837515 \mathrm{MeV}, \quad m_{v_{\mu}}=0 \mathrm{MeV}, \\
m_{\tau} & =1776.82 \mathrm{MeV}, \quad m_{v_{\tau}}=0 \mathrm{MeV}, \\
m_{u} & =68.7 \mathrm{MeV}, \quad m_{d}=68.7 \mathrm{MeV}, \\
m_{c} & =1.275 \mathrm{GeV}, \quad m_{s}=95.0 \mathrm{MeV}, \\
m_{t} & =173.07 \mathrm{GeV}, \quad m_{b}=4.18 \mathrm{GeV} .
\end{aligned}
$$

According to Ref. [66], $m_{s}$ is an estimate of a so-called "current quark mass" in the $\overline{\mathrm{MS}}$ scheme at the scale $\mu \approx 2 \mathrm{GeV} . m_{c} \equiv m_{c}\left(m_{c}\right)$ and $m_{b} \equiv m_{b}\left(m_{b}\right)$ are the "running" masses in the $\overline{\mathrm{MS}}$ scheme. The top quark mass as well as the lepton masses are defined OS. $m_{u}$ and $m_{d}$ are effective parameters, calculated through the hadronic contributions to

$$
\begin{aligned}
\Delta \alpha_{\mathrm{had}}^{(5)}\left(M_{Z}\right) & =\frac{\alpha}{\pi} \sum_{f=u, c, d, s, b} Q_{f}^{2}\left(\ln \frac{M_{Z}^{2}}{m_{f}^{2}}-\frac{5}{3}\right) \\
& \sim 0.027723 .
\end{aligned}
$$

- The CKM matrix has been set to unity.

- Gauge-boson masses:

$$
M_{Z}=91.1876 \mathrm{GeV}, \quad M_{W}=80.385 \mathrm{GeV} .
$$

- Coupling constants:

$\alpha(0)=1 / 137.0359895, \quad \alpha_{s}\left(M_{Z}\right)=0.1184$,

where the running and decoupling of $\alpha_{s}$ is described in Sect. 2.5.

The Higgs sector quantities (masses, mixings, etc.) have been evaluated using FeynHiggs (version 2.10.2) [33-38]. 
Table 2 MSSM parameters for the initial numerical investigation; all parameters (except of $t_{\beta}$ ) are in GeV. In our analysis $M_{\tilde{Q}_{3}}, M_{\tilde{U}_{3}}, M_{\tilde{D}_{3}}$, $M_{\tilde{L}_{3}}$, and $M_{\tilde{E}_{3}}$ are chosen such that the values of $m_{\tilde{t}_{1}}, m_{\tilde{t}_{2}}, m_{\tilde{b}_{2}}, m_{\tilde{v}_{\tau}}$, and $m_{\tilde{\tau}_{2}}$ are realized. For the $\tilde{\mathrm{d}}_{g}$ and $\tilde{\mathrm{e}}_{g}$ sector the shifts in $M_{\tilde{Q}, \tilde{D}}\left(\tilde{\mathrm{d}}_{g}\right)$ and $M_{\tilde{L}, \tilde{E}}\left(\tilde{\mathrm{e}}_{g}\right)$ as defined in Eqs. (12) and (14) are taken into account,

\begin{tabular}{|c|c|c|c|c|c|c|c|c|c|c|c|c|c|}
\hline Scen. & $t_{\beta}$ & $m_{\tilde{t}_{1}}$ & $m_{\tilde{t}_{2}}$ & $m_{\tilde{b}_{2}}$ & $m_{\tilde{v}_{\tau}}$ & $m_{\tilde{\tau}_{2}}$ & $\mu$ & $\left|A_{t}\right|$ & $\left|A_{b}\right|$ & $\left|A_{\tau}\right|$ & $M_{1}$ & $M_{2}$ & $M_{3}$ \\
\hline $\mathrm{S} 1 / \mathrm{S} 2 / \mathrm{S} 3$ & 10 & 394 & 771 & 582 & 280 & 309 & 500 & 1200 & 600 & 1000 & 300 & 600 & 1500 \\
\hline Scen. & $M_{H^{ \pm}}$ & $m_{h_{1}}$ & $m_{h_{2}}$ & $m_{h_{3}}$ & $m_{\tilde{\tau}_{1}}$ & $m_{\tilde{b}_{1}}$ & & & & & & & \\
\hline S1 & 1000 & 123.405 & 996.766 & 996.813 & 282.517 & 513.2 & & & & & & & \\
\hline S2 & 1400 & 123.428 & 1397.299 & 1398.596 & 282.337 & 513. & & & & & & & \\
\hline S3 & 1600 & 123.436 & 1597.174 & 1597.524 & 282.265 & 513. & & & & & & & \\
\hline
\end{tabular}

concerning $m_{\tilde{\tau}_{1}}$ and $m_{\tilde{b}_{1}}$ (rounded to $1 \mathrm{MeV}$ ). The values for $A_{t}, A_{b}$, and $A_{\tau}$ are chosen such that charge- and/or color-breaking minima are avoided [90-96]. It should be noted that for the first and second generation of sfermions we chose instead $M_{\tilde{L}, \tilde{E}}=1500 \mathrm{GeV}$ and $M_{\tilde{Q}, \tilde{U}, \tilde{D}}=2000 \mathrm{GeV}$

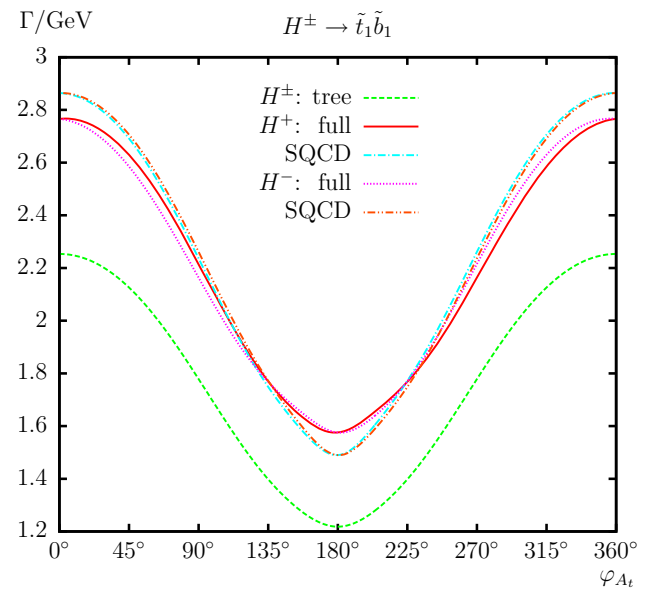

Fig. $3 \Gamma\left(H^{ \pm} \rightarrow \tilde{t}_{1} \tilde{b}_{1}\right)$. Tree-level, full, and SQCD one-loop corrected partial decay widths are shown. The upper plot shows the partial decay width with $M_{H^{ \pm}}$varied. The lower plots show the complex phases $\varphi_{A_{t}}$

Furthermore, the current limits for SUSY particle searches [66] are roughly fulfilled in our analysis.

We emphasize again that the analytical calculation has been done for all decays into sfermions, but in the numerical analysis we concentrate on the decays to third generation sfermions. Results are shown for some representative numerical examples. The parameters are chosen according

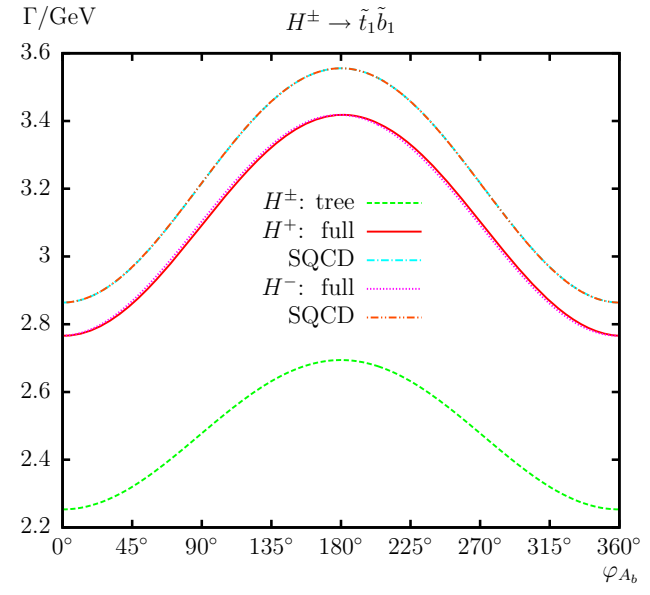

(left) and $\varphi_{A_{b}}$ (right) varied with parameters chosen according to S1 (see Table 2)

to the scenarios, S1, S2 and S3, shown in Table 2. The scenarios are defined such that a maximum number of (third generation) decay modes are open simultaneously to permit an analysis of all channels, i.e. not picking specific parameters for each decay. For the same reason we do not demand that the lightest Higgs boson has a mass around $\sim 125 \mathrm{GeV}$, although for most of the parameter space this is given. The 


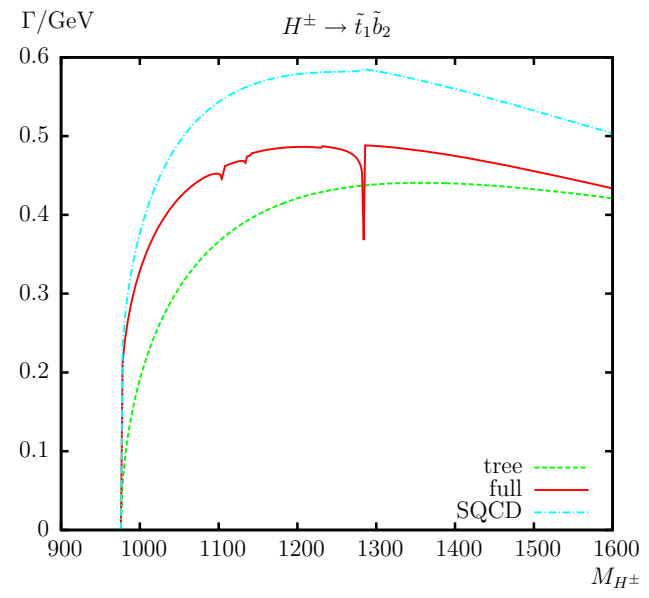

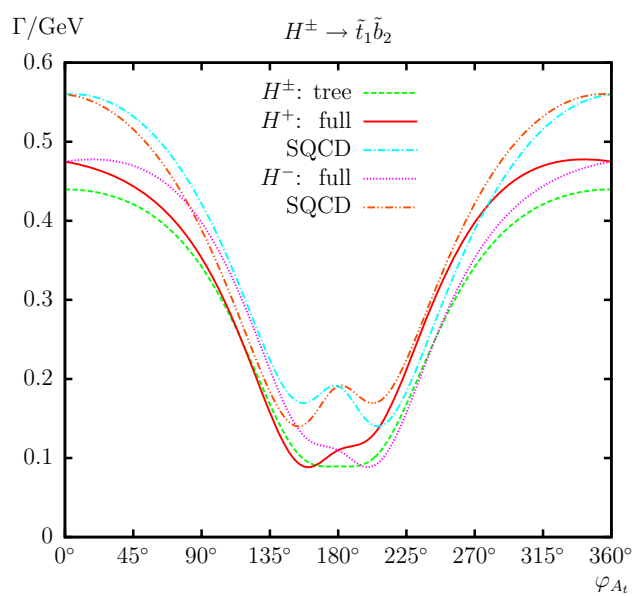

Fig. $4 \Gamma\left(H^{ \pm} \rightarrow \tilde{t}_{1} \tilde{b}_{2}\right)$. Tree-level, full, and SQCD one-loop corrected partial decay widths are shown. The upper plot shows the partial decay width with $M_{H^{ \pm}}$varied. The lower plots show the complex phases $\varphi_{A_{t}}$

only parameter varied with a sizable impact on $m_{h_{1}}$ is $\varphi_{A_{t}}$. For $110^{\circ}<\varphi_{A_{t}}<250^{\circ}$ the light Higgs-boson mass drops slightly below $122 \mathrm{GeV}$, but stays above $121 \mathrm{GeV}$, where a theory uncertainty in the prediction of $\sim 3 \mathrm{GeV}$ [37] must be kept in mind.

The numerical results we will show in the next subsections are of course dependent on choice of the SUSY parameters. Nevertheless, they give an idea of the relevance of the full one-loop corrections. Channels (and their respective one-loop corrections) that may look unobservable due to the smallness of their decay width in the plots shown below, could become important if other channels are kinematically forbidden.

\subsection{Full one-loop results for varying $\boldsymbol{M}_{\boldsymbol{H}^{ \pm}}$and $\boldsymbol{\varphi}_{\boldsymbol{A}}$}

The results shown in this and the following subsections consist of "tree", which denotes the tree-level value and of "full", which is the partial decay width including all oneloop corrections as described in Sect. 3. Also shown are the

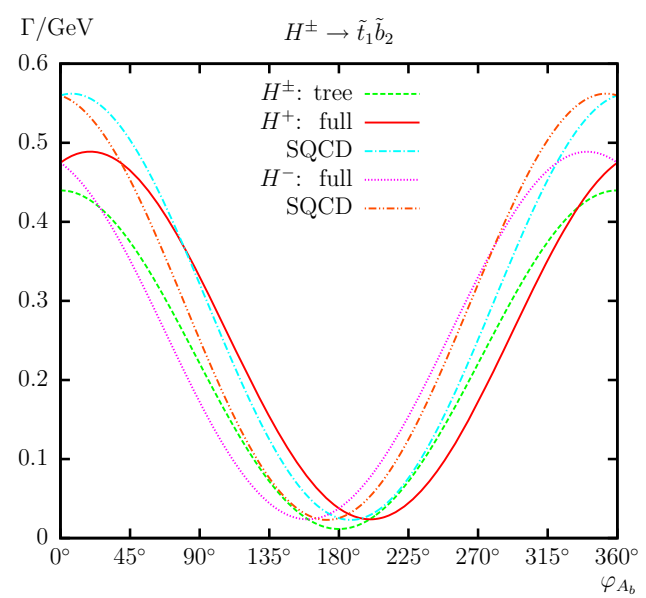

(left) and $\varphi_{A_{b}}$ (right) varied with parameters chosen according to S2 (see Table 2)

pure SUSY-QCD one-loop corrections (“SQCD”) for colored decays. We restrict ourselves to the analysis of the decay widths themselves, since the one-loop effects on the branching ratios are strongly parameter dependent, as discussed in the previous subsection.

When performing an analysis involving complex parameters it should be noted that the results for physical observables are affected only by certain combinations of the complex phases of the parameters $\mu$, the trilinear couplings $A_{t, b, \tau}$ and the gaugino mass parameters $M_{1,2,3}$ [97,98]. It is possible, for instance, to rotate the phase $\varphi_{M_{2}}$ away. Experimental constraints on the (combinations of) complex phases arise, in particular, from their contributions to electric dipole moments of the electron and the neutron (see Refs. [99-101] and references therein), of the deuteron [102] and of heavy quarks [103,104]. While SM contributions enter only at the threeloop level, due to its complex phases the MSSM can contribute already at one-loop order. Large phases in the first two generations of sfermions can only be accommodated if these generations are assumed to be very heavy $[105,106]$ or large 


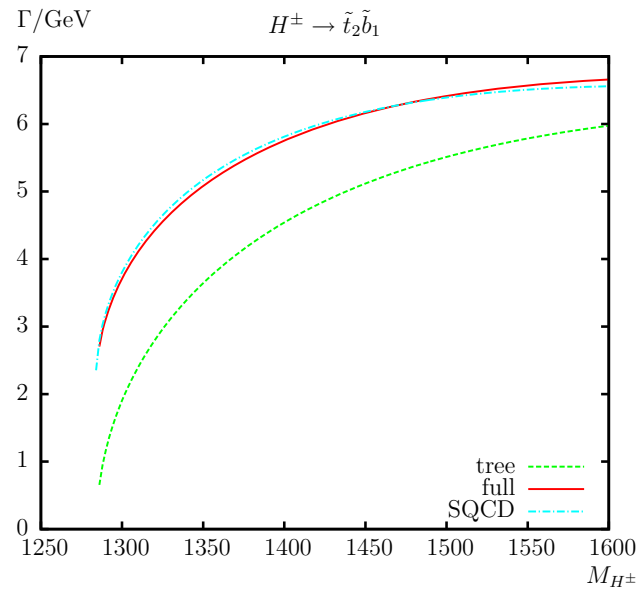

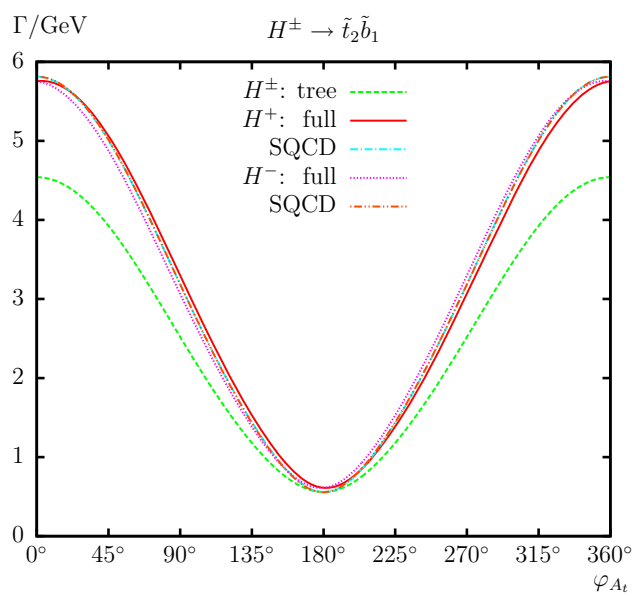

Fig. $5 \Gamma\left(H^{ \pm} \rightarrow \tilde{t}_{2} \tilde{b}_{1}\right)$. Tree-level, full, and SQCD one-loop corrected partial decay widths are shown. The upper plot shows the partial decay width with $M_{H^{ \pm}}$varied. The lower plots show the complex phases $\varphi_{A_{t}}$

cancelations occur [107-109]; see, however, the discussion in Ref. [110]. A review can be found in Ref. [111]. Accordingly (using the convention that $\varphi_{M_{2}}=0$, as done in this paper), in particular, the phase $\varphi_{\mu}$ is tightly constrained [112], while the bounds on the phases of the third generation trilinear couplings are much weaker. Setting $\varphi_{\mu}=\varphi_{M_{1}}=\varphi_{\tilde{g}}=0$ leaves us with $\varphi_{A_{t}}, \varphi_{A_{b}}$, and $\varphi_{A_{\tau}}$ as the only complex valued parameters. It should be noted that the tree-level prediction depends on $\varphi_{A}$ via the sfermion mixing matrix and the variation of the phases $\varphi_{A_{t, b}}\left(\varphi_{A_{\tau}}\right)$ leads to a maximal change of $10 \%$ $(20 \%)$ in the squark (slepton) masses.

Since now complex trilinear $A_{f}$ parameters can appear in the couplings, contributions from absorptive parts of selfenergy type corrections on external legs can arise. The corresponding formulas for an inclusion of these absorptive contributions via finite wave function correction factors can be found in $[53,57]$.

We start the numerical analysis with partial decay widths of $H^{ \pm}$evaluated as a function of $M_{H^{ \pm}}$, starting at $M_{H^{ \pm}}=$

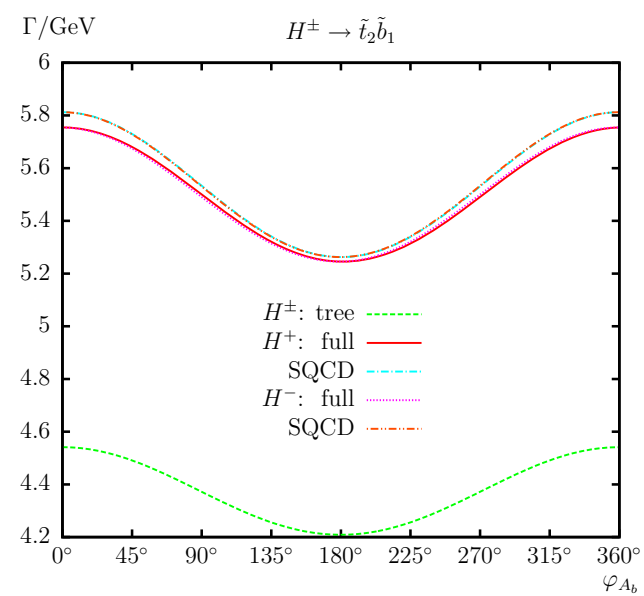

(left) and $\varphi_{A_{b}}$ (right) varied with parameters chosen according to S2 (see Table 2)

$600 \mathrm{GeV}$ up to $M_{H^{ \pm}}=1.6 \mathrm{TeV}$, which roughly coincides with the reach of the LHC for high-luminosity running as well as an $e^{+} e^{-}$collider with a center-of-mass energy up to $\sqrt{s} \sim$ $3 \mathrm{TeV}[15,16]$. Then we turn to the $h_{n}(n=2,3)$ decays.

\subsection{1 $\boldsymbol{H}^{ \pm}$decays into sfermions}

In Figs. 3, 4, 5, 6, 7 and 8 we show the results for the processes $H^{ \pm} \rightarrow \tilde{f}_{i} \tilde{f}_{j}^{\prime}(i, j=1,2)$ as a function of $M_{H^{ \pm}}$and as a function of the relevant complex phases $\varphi_{A}$. These are of particular interest for LHC analyses $[113,114]$ (as emphasized in Sect. 1). The various visible (or hardly visible) dips and steps occurring for different values of $M_{H^{ \pm}}$in the plots are summarized in Table 3, labeled TC1 to TC7.

We start with the decay $H^{ \pm} \rightarrow \tilde{t}_{1} \tilde{b}_{1}$. In the upper plot of Fig. 3 the dips/thresholds TC3, TC4, TC5, TC6, and TC7 appear; see Table 3 . The size of the corrections of the partial decay widths is especially large very close to the production 


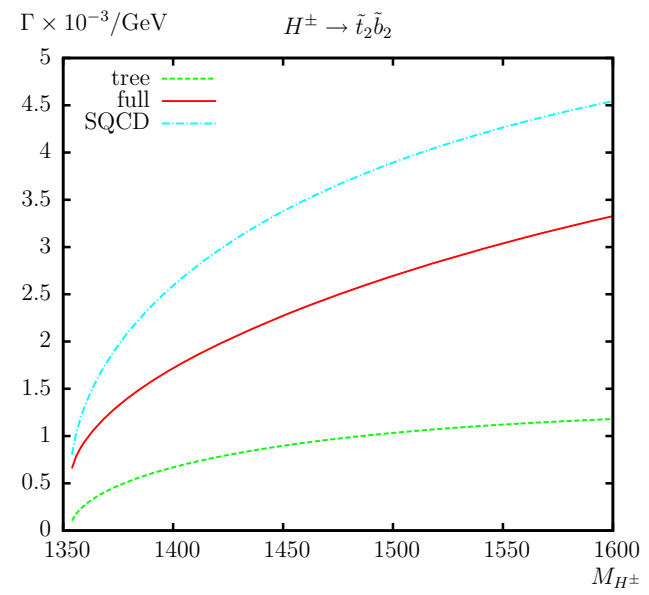

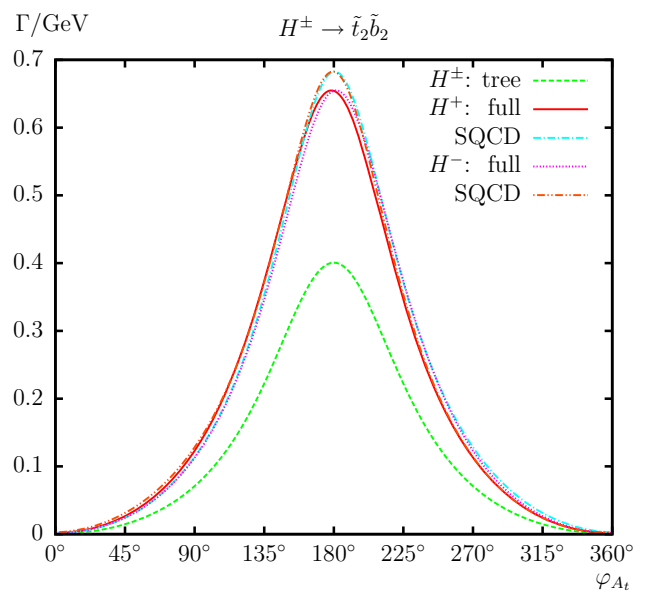

Fig. $6 \Gamma\left(H^{ \pm} \rightarrow \tilde{t}_{2} \tilde{b}_{2}\right)$. Tree-level, full, and SQCD one-loop corrected partial decay widths are shown. The upper plot shows the partial decay width with $M_{H^{ \pm}}$varied. The lower plots show the complex phases $\varphi_{A_{t}}$

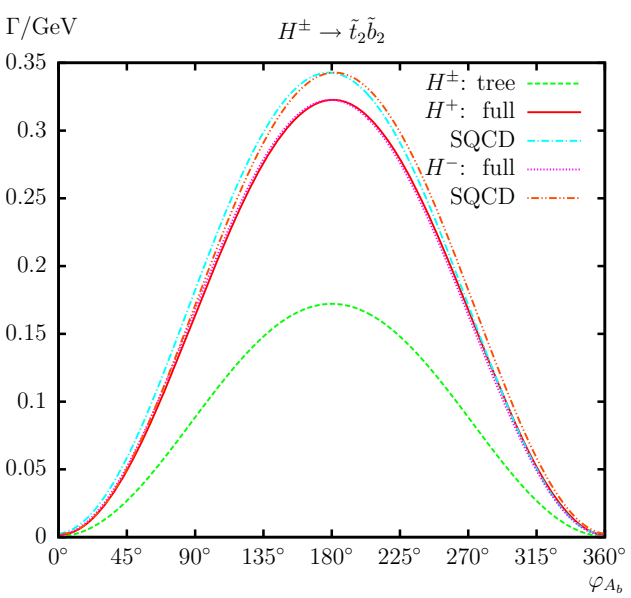

(left) and $\varphi_{A_{b}}$ (right) varied with parameters chosen according to S2 (see Table 2)
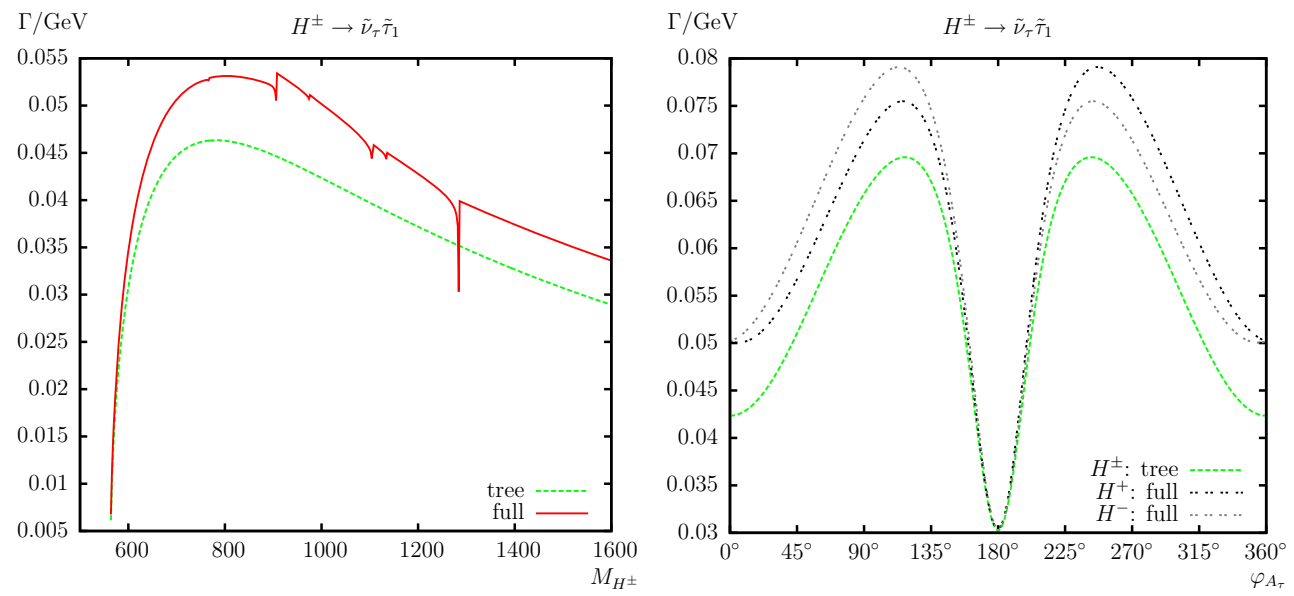

Fig. $7 \Gamma\left(H^{ \pm} \rightarrow \tilde{v}_{\tau} \tilde{\tau}_{1}\right)$. Tree-level and full one-loop corrected partial decay widths are shown. The left plot shows the partial decay width with $M_{H^{ \pm}}$varied; the right plot shows the complex phase $\varphi_{A_{\tau}}$ varied with parameters chosen according to S1 (see Table 2) 

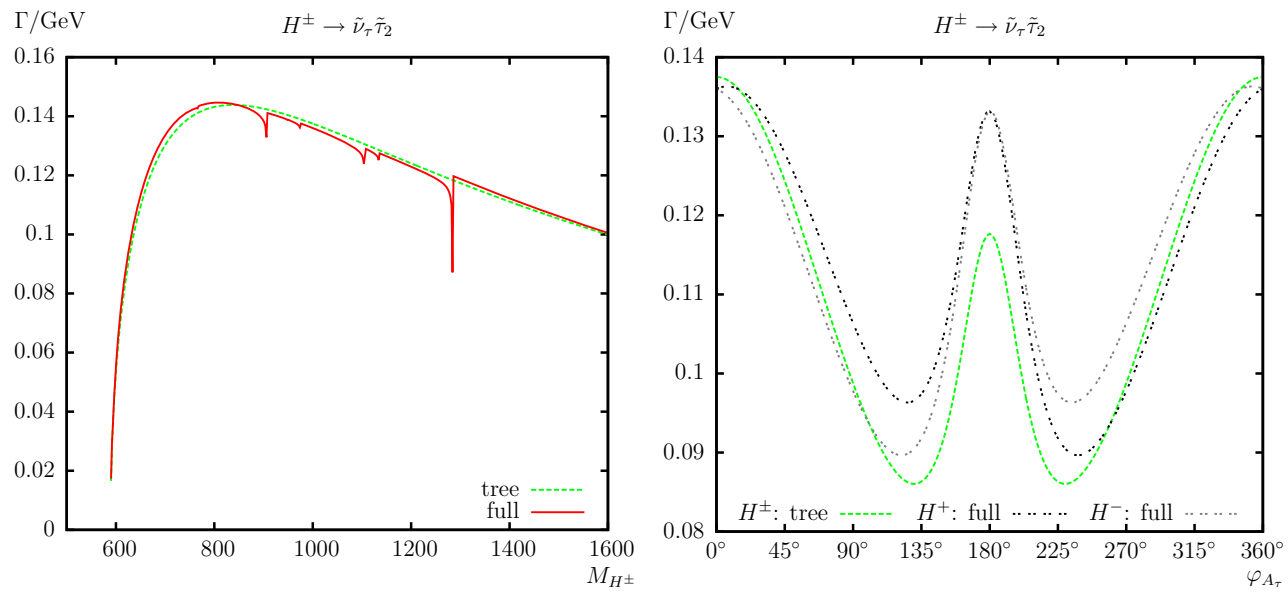

Fig. $8 \Gamma\left(H^{ \pm} \rightarrow \tilde{v}_{\tau} \tilde{\tau}_{2}\right)$. Tree-level and full one-loop corrected partial decay widths are shown. The left plot shows the partial decay width with $M_{H^{ \pm}}$varied; the right plot shows the complex phase $\varphi_{A_{\tau}}$ varied with parameters chosen according to S1 (see Table 2)

Table 3 Thresholds in charged Higgs-boson decays

\begin{tabular}{lll}
\hline TC1: & $M_{H^{ \pm}} \approx 768 \mathrm{GeV}$ & $m_{\tilde{\chi}_{1}^{ \pm}}+m_{\tilde{\chi}_{1}^{0}}=M_{H^{ \pm}}$ \\
TC2: & $M_{H^{ \pm}} \approx 907 \mathrm{GeV}$ & $m_{\tilde{t}_{1}}+m_{\tilde{b}_{1}}=M_{H^{ \pm}}$ \\
TC3: & $M_{H^{ \pm}}=976 \mathrm{GeV}$ & $m_{\tilde{t}_{1}}+m_{\tilde{b}_{2}}=M_{H^{ \pm}}$ \\
TC4: & $M_{H^{ \pm}} \approx 1105 \mathrm{GeV}$ & $m_{\tilde{\chi}_{1}^{ \pm}}+m_{\tilde{\chi}_{4}^{0}}=M_{H^{ \pm}}$ \\
TC5: & $M_{H^{ \pm}} \approx 1108 \mathrm{GeV}$ & $m_{\tilde{\chi}_{2}^{ \pm}}+m_{\tilde{\chi}_{2}^{0}}=M_{H^{ \pm}}$ \\
TC6: & $M_{H^{ \pm}} \approx 1135 \mathrm{GeV}$ & $m_{\tilde{\chi}_{2}^{ \pm}}+m_{\tilde{\chi}_{3}^{0}}=M_{H^{ \pm}}$ \\
TC7: & $M_{H^{ \pm}} \approx 1284 \mathrm{GeV}$ & $m_{\tilde{t}_{2}}+m_{\tilde{b}_{1}}=M_{H^{ \pm}}$ \\
\hline
\end{tabular}

threshold $^{11}$ from which on the considered decay mode is kinematically possible. Away from this production threshold relative corrections of $\sim+23 \%$ are found in S1 (see Table 2), of $\sim+5 \%$ in S2 and of $\sim+3 \%$ in S3. The SQCD corrections are slightly larger, i.e. the EW corrections reduce the overall size of the loop corrections by $\sim 17 \%$.

In the lower plots of Fig. 3 we show the complex phases $\varphi_{A_{t, b}}$ varied at $M_{H^{ \pm}}=1000 \mathrm{GeV}$. The tree-level dependence on the two phases is very different. While for negative $A_{t}$ a reduction by nearly $50 \%$ w.r.t. positive $A_{t}$ is found, negative $A_{b}$ leads to an enhancement of about $25 \%$. The full corrections with $\varphi_{A_{t}}$ varied are up to $\sim+29 \%$ with slightly larger or lower values for the SQCD corrections by up to $\sim \pm 4 \%$. The asymmetry depending on $\varphi_{A_{t}}$ is rather small. $\varphi_{A_{b}}$ varied can reach $\sim+27 \%$ with slightly larger values for the SQCD corrections $\sim+31 \%$. Here the $\varphi_{A_{b}}$ asymmetry is hardly visible.

In Fig. 4 we show the results for $H^{ \pm} \rightarrow \tilde{t}_{1} \tilde{b}_{2}$. In the upper plot the production threshold matches exactly $m_{\tilde{t}_{1}}+m_{\tilde{b}_{2}}=$

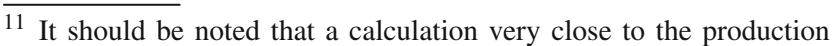
threshold requires the inclusion of additional (nonrelativistic) contributions, which is beyond the scope of this paper. Consequently, very close to the production threshold our calculation (at the tree and loop level) does not provide a very accurate description of the decay width.
}

$M_{H^{ \pm}}=976 \mathrm{GeV}$. This is due to the fact that all three masses are input parameters (i.e. integers; see Table 2) and our step size for $M_{H^{ \pm}}$is $2 \mathrm{GeV}$. Therefore the decay width starts "accidentally" exactly from zero. The dips/thresholds appearing are TC4, TC5, TC6, and TC7. Relative corrections of $\sim+8 \%$ are found at $M_{H^{ \pm}}=1400 \mathrm{GeV}$ in S2 (see Table 2) (and $\sim+3 \%$ at $M_{H^{ \pm}}=1600 \mathrm{GeV}$ in S3). The SQCD corrections alone would lead to an increase of $\sim+21 \%$ in S2 $(\sim+13 \%$ in S3), i.e. they overestimate the full corrections by roughly a factor of three.

In the lower plots of Fig. 4 the results are shown for S2 as a function of $\varphi_{A_{t, b}}$. One can see that the size of the corrections to the partial decay width vary substantially with the complex phases $\varphi_{A_{t, b}}$ at $M_{H^{ \pm}}=1400 \mathrm{GeV}$. In case of $H^{+} \rightarrow \tilde{t}_{1} \tilde{b}_{2}$ (lower left plot) the behavior in the region $135^{\circ}<\varphi_{A_{t}}<225^{\circ}$ is due to an accidental interplay of the shifted sbottom mixing angle $U_{21}^{\tilde{b} *}$ multiplied with $A_{t} U_{12}^{\tilde{t}}$, already present in the tree coupling. (In the $H^{-}$decay it is complex conjugated: $U_{21}^{\tilde{b}} A_{t}^{*} U_{12}^{\tilde{t} *}$.) Furthermore, effects from absorptive contributions also play an important role for the (rather large) asymmetries. The largest contribution to the asymmetries in the lower right plot stems from the $\tilde{t}_{1}-\tilde{b}_{2}-\gamma$ vertex. At $\varphi_{A_{t}}=180^{\circ}$ the full corrections reach $\sim+23 \%$, while the SQCD corrections are much larger $\sim+77 \%$. At $\varphi_{A_{b}}=90^{\circ}$ the $H^{+}\left(H^{-}\right)$full corrections reach $\sim+55 \%(\sim-22 \%)$, while the SQCD corrections are $\sim+38 \%(\sim+8 \%) .^{12}$

Next, in Fig. 5 the results for $H^{ \pm} \rightarrow \tilde{t}_{2} \tilde{b}_{1}$ are displayed. In the upper plot the results are shown as a function of $M_{H^{ \pm}}$. Relative corrections of $\sim+27 \%$ are found at

\footnotetext{
12 It should be noted that at $\varphi_{A_{b}} \approx 180^{\circ}$ the (positive) loop corrections can be larger then the tree results because there the tree-level decay width is accidentally small; see the lower right plot of Fig. 4. In case of large negative loop corrections we will add $\left|\mathcal{M}_{1 \text {-loop }}\right|^{2}$ to obtain, at least, a positive decay width (see below).
} 


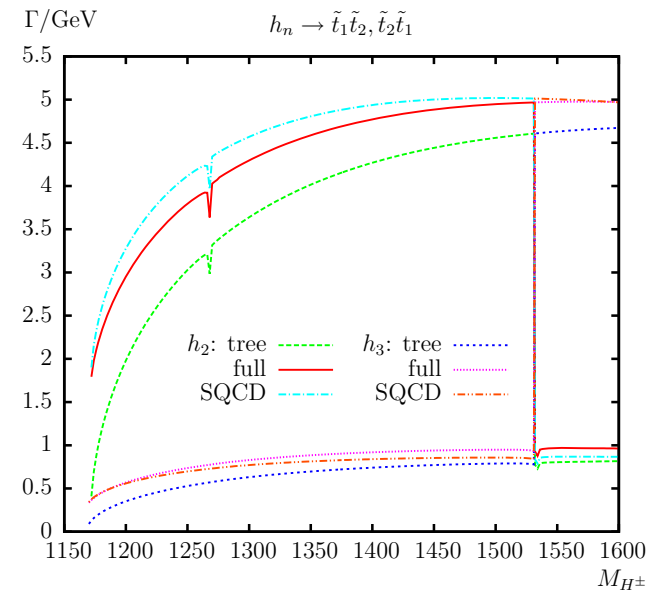

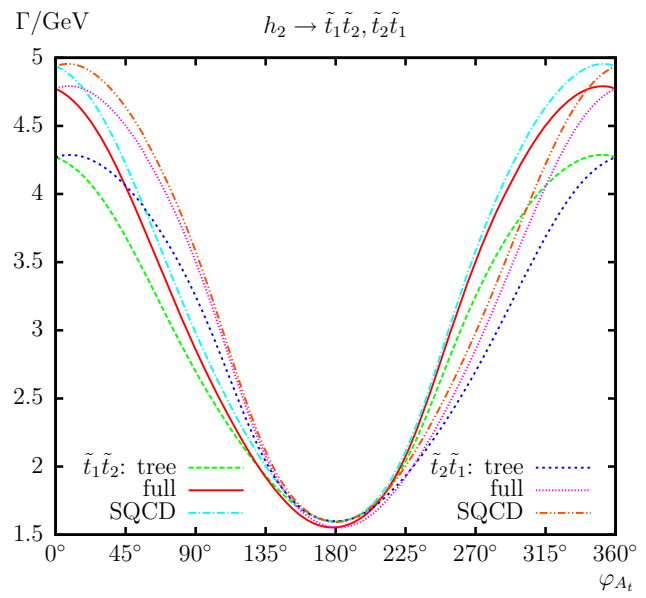

Fig. $9 \Gamma\left(h_{n} \rightarrow \tilde{t}_{1} \tilde{t}_{2}, \tilde{t}_{2} \tilde{t}_{1}\right)$. Tree-level, full, and SQCD one-loop corrected partial decay widths are shown. The upper plot shows the partial decay width with $M_{H^{ \pm}}$varied; the lower plots show the complex phase

$M_{H^{ \pm}}=1400 \mathrm{GeV}$ (see Table 2 ). In this case the EW corrections hardly contribute to the overall one-loop contribution.

In the lower plots the results are displayed as a function of $\varphi_{A_{t, b}}$ in S2. In the left plot one can see that the size of the corrections to the partial decay width vary substantially with the complex phase $\varphi_{A_{t}}$. For all $\varphi_{A_{t}}$ the full and SQCD corrections are of similar size and deviate between $+9 \%$ and $+27 \%$. The same holds for $\varphi_{A_{b}}$ with small differences between the full and SQCD corrections, which vary only between $+25 \%$ and $+27 \%$. Here the asymmetries are small and hardly visible.

The decay $H^{ \pm} \rightarrow \tilde{t}_{2} \tilde{b}_{2}$ is shown in Fig. 6. The overall size of this decay width (with real phases) is (accidentally) very small around $\sim 2 \times 10^{-3} \mathrm{GeV}$. Consequently, the loop corrections, as shown in the upper plot, can be larger than the tree-level result. The SQCD corrections alone would overestimate the full result by about $\sim 50 \%$.

In the lower plots of Fig. 6 one can see that the size of the tree-level result depends again strongly on the two

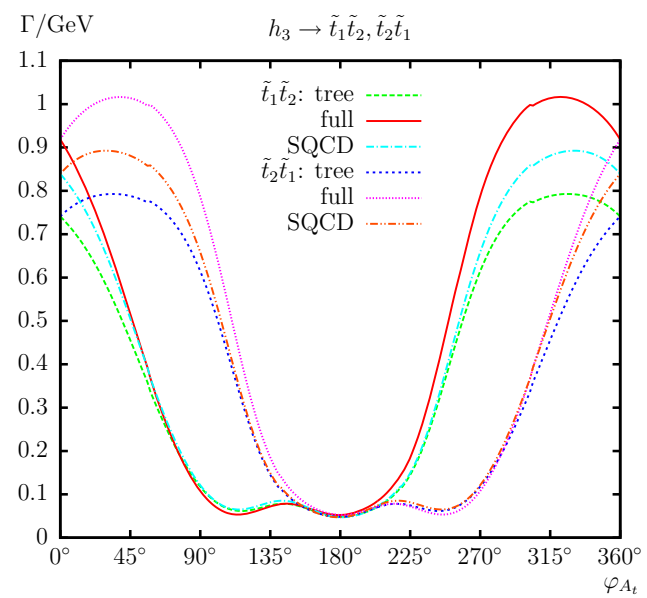

$\varphi_{A_{t}}$ varied for $h_{2}$ decays (left) and $h_{3}$ decays (right) with parameters chosen according to $\mathrm{S} 2$ (see Table 2)

phases. Values of $\sim 0.4(0.16) \mathrm{GeV}$ are reached for negative $\varphi_{A_{t}}\left(\varphi_{A_{b}}\right)$. Again the loop corrections can be substantial. At $\varphi_{A_{t}}=180^{\circ}$ the full corrections reach $\sim+63 \%$, while the SQCD corrections are larger $\sim+72 \%$. At $\varphi_{A_{b}}=180^{\circ}$ the full corrections reach $\sim+87 \%$, while the SQCD corrections are up to $\sim+90 \%$. The asymmetries are found to be rather small due to accidentally cancelations with absorptive contributions.

Now we turn to the charged Higgs-boson decays to scalar leptons, $H^{ \pm} \rightarrow \tilde{v}_{\tau} \tilde{\tau}_{1}$ in Fig. 7 and $H^{ \pm} \rightarrow \tilde{v}_{\tau} \tilde{\tau}_{2}$ in Fig. 8 . The left plots show the results as a function of $M_{H^{ \pm}}$, while the right plots analyze the dependence on $\varphi_{A_{\tau}}$ for $M_{H^{ \pm}}=$ $1000 \mathrm{GeV}$. In the left plots all seven thresholds, given in Table 3, appear as dips. At $M_{H^{ \pm}}=1000 \mathrm{GeV}$ one-loop corrections of $\sim+19 \%$ are found for $H^{ \pm} \rightarrow \tilde{v}_{\tau} \tilde{\tau}_{1}$, while for $H^{ \pm} \rightarrow \tilde{\nu}_{\tau} \tilde{\tau}_{2}$ they are only $\sim-1 \%$.

The maximum values of the full one-loop corrections as a function of $\varphi_{A_{\tau}}$ reach $\sim+15 \%(+13 \%)$ for $H^{ \pm} \rightarrow$ $\tilde{v}_{\tau} \tilde{\tau}_{1}\left(\tilde{v}_{\tau} \tilde{\tau}_{2}\right)$. The asymmetries in the decays of a negative 


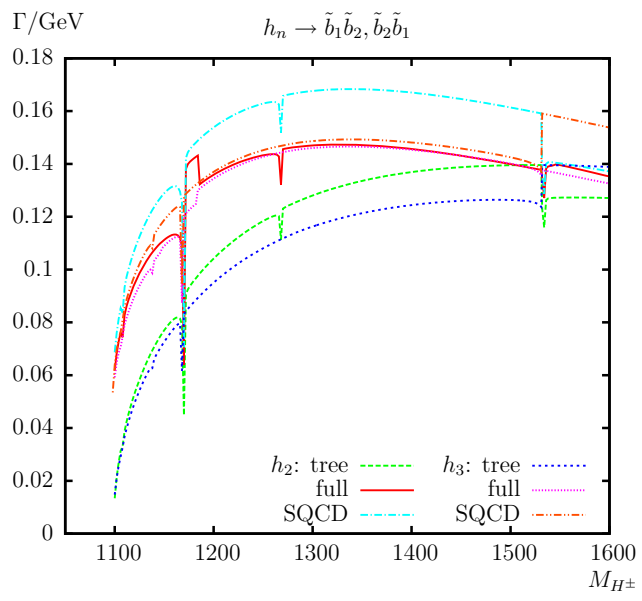

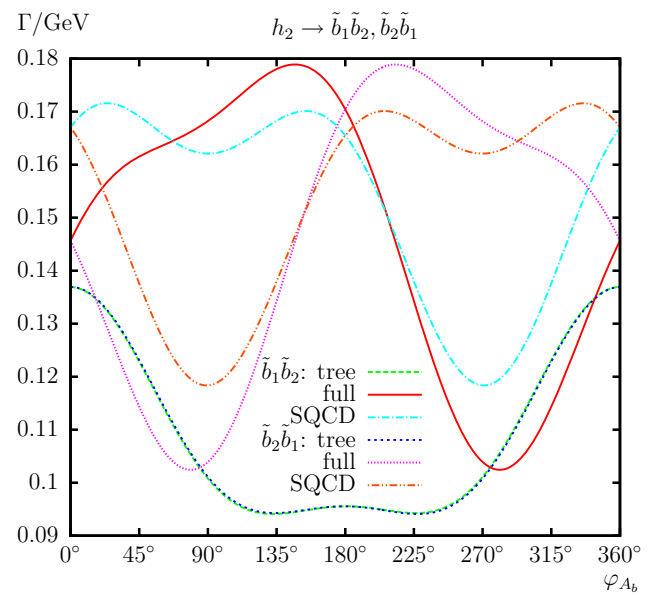

Fig. $10 \Gamma\left(h_{n} \rightarrow \tilde{b}_{1} \tilde{b}_{2}, \tilde{b}_{2} \tilde{b}_{1}\right)$. Tree-level, full, and SQCD one-loop corrected partial decay widths are shown. The upper plot shows the partial decay width with $M_{H^{ \pm}}$varied; the lower plots show the com-

charged Higgs w.r.t. a positively charged Higgs are substantial. The size of the respective loop corrections can nearly be twice as large in one case w.r.t. to the other, depending whether $\varphi_{A_{\tau}} \leq 180^{\circ}$ or $\varphi_{A_{\tau}} \geq 180^{\circ}$ is considered.

\subsection{2 $\boldsymbol{h}_{\boldsymbol{n}}$ decays into sfermions}

We now turn to the decay modes $h_{n} \rightarrow \tilde{f}_{i} \tilde{f}_{j}(n=2,3 ; i, j=$ 1,2). Results are shown in the Figs. 9, 10, 11, 12, 13, 14, 15, 16, 17 and 18.

Before discussing every figure in detail, it should be noted that there is a subtleness concerning the mixture of the $h_{n}$ bosons. Depending on the input parameters, the higher-order corrections to the three neutral Higgs-boson masses can vary substantially. The mass ordering $m_{h_{1}}<m_{h_{2}}<m_{h_{3}}$ (as performed automatically by FeynHiggs), even in the case of real parameters, can yield a heavy $\mathcal{C P}$-even Higgs mass higher or lower than the (heavy) $\mathcal{C P}$-odd Higgs mass. Such a

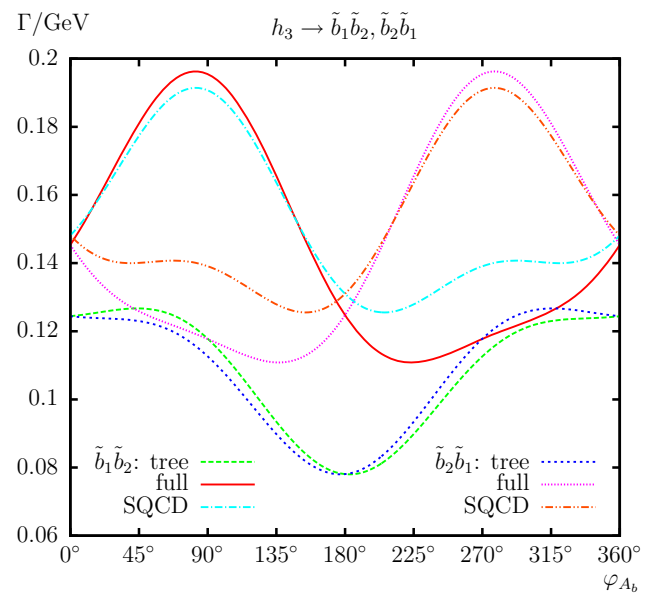

plex phase $\varphi_{A_{b}}$ varied for $h_{2}$ decays (left) and $h_{3}$ decays (right) with parameters chosen according to S2 (see Table 2)

transition in the mass ordering (or "mass crossing") is accompanied by an abrupt change in the Higgs mixing matrix $\hat{\mathbf{Z}}{ }^{13}$ For our input parameters (see Table 2) there are two (possible) crossings. The first (called "MC1" below) appears at $M_{H^{ \pm}} \approx 1006 \mathrm{GeV}$. Before the crossing we find $h_{2} \sim H$ $\left(h_{3} \sim A\right)$, whereas after the crossing it changes to $h_{2} \sim A$ $\left(h_{3} \sim H\right)$. The second crossing (called "MC2") is found at $M_{H^{ \pm}} \approx 1532 \mathrm{GeV}$, i.e. the changing of the mixture from $h_{2} \sim A\left(h_{3} \sim H\right)$ to $h_{2} \sim H\left(h_{3} \sim A\right)$. Very close to the mass crossings the $\hat{\mathbf{Z}}$ matrix can yield small numerical instabilities. As an example, for $1532 \mathrm{GeV} \lesssim M_{H^{ \pm}} \lesssim 1536 \mathrm{GeV}$ the $\hat{\mathbf{Z}}$ matrix causes structures appearing similar to "usual" dips from thresholds. All the dips/thresholds (some are hardly

\footnotetext{
${ }^{13}$ In our case the $Z$-factor matrix $\hat{Z}_{i j} \equiv \operatorname{ZHiggs}[i, j]$; see Ref. [57] (and Ref. [33]), which contributes at tree level. Furthermore, $\hat{\mathbf{Z}}$ is calculated by FeynHiggs, which uses $m_{b}\left(m_{b}\right)$ and tree-level sfermion masses instead of the shifted masses, causing a slight displacement in the threshold position.
} 


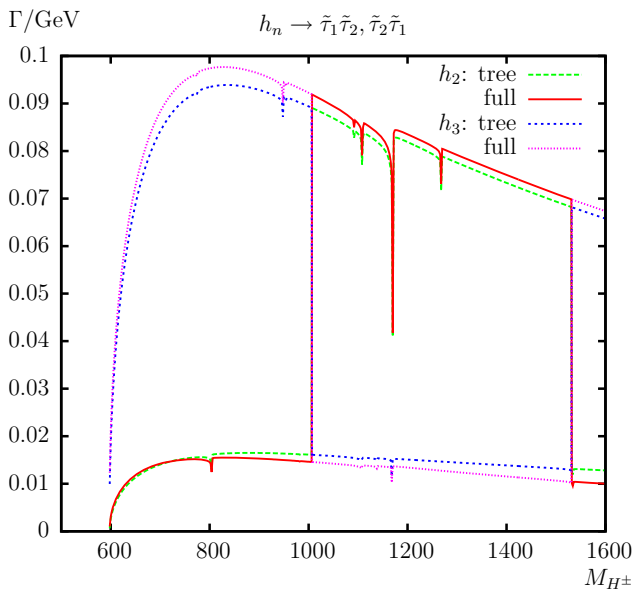

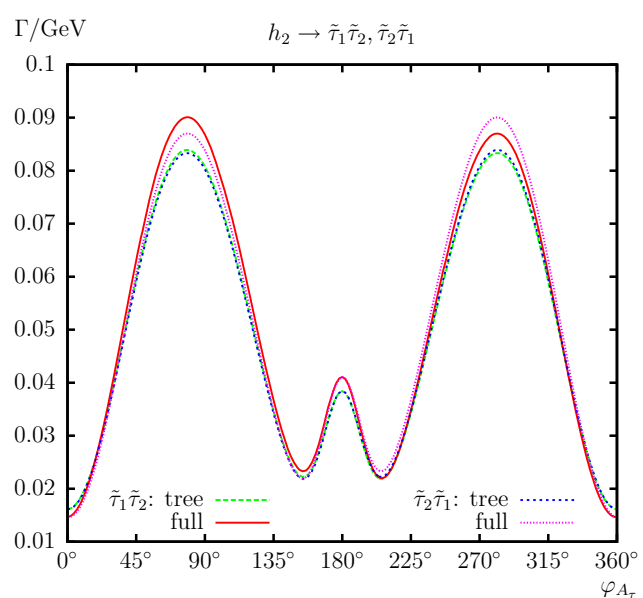

Fig. $11 \Gamma\left(h_{n} \rightarrow \tilde{\tau}_{1} \tilde{\tau}_{2}, \tilde{\tau}_{2} \tilde{\tau}_{1}\right)$. Tree-level and full one-loop corrected partial decay widths are shown. The upper plot shows the partial decay width with $M_{H^{ \pm}}$varied; the lower plots

visible) appearing in the figures are listed in Table 4, labeled as TN1 to TN17.

We start with the decay $h_{n} \rightarrow \tilde{t}_{1} \tilde{t}_{2}, \tilde{t}_{2} \tilde{t}_{1}$ as shown in Fig. 9 . The upper plot shows the results as a function of $M_{H^{ \pm}}$, whereas in the lower plots we present the decay widths as a function of $\varphi_{A_{t}}$ in S2. We show separately the results for the $h_{2}$ and $h_{3}$ decay widths. In the upper plot of Fig. 9 the dips/thresholds TN16 and TN17 appear. Away from the production threshold relative corrections of $\sim+12 \%$ are found in S2 (see Table 2) for the $h_{2}$ decay. There the SQCD corrections overestimate the full correction by about $30 \%$. In case of the $h_{3}$ decay the relative corrections are $\sim+24 \%$ in S2 (see Table 2) and the SQCD corrections underestimate the full result by about $50 \%$. The MC2 can be observed at $M_{H^{ \pm}} \sim 1532 \mathrm{GeV}$ as described above. Here $h_{2}$ and $h_{3}$ change their role. Within the unrotated scalar top basis the $\mathcal{C P}$-odd Higgs boson can only decay as $A \rightarrow \tilde{t}_{L} \tilde{t}_{R}, \tilde{t}_{R} \tilde{t}_{L}$, but not as $A \rightarrow \tilde{t}_{L} \tilde{t}_{L}, \tilde{t}_{R} \tilde{t}_{R}$, whereas the $\mathcal{C} \mathcal{P}$-even Higgs boson has all four decays possible. Consequently, the decay to $\tilde{t}_{1} \tilde{t}_{2}, \tilde{t}_{2} \tilde{t}_{1}$ can depend strongly on the $\mathcal{C P}$ nature of the

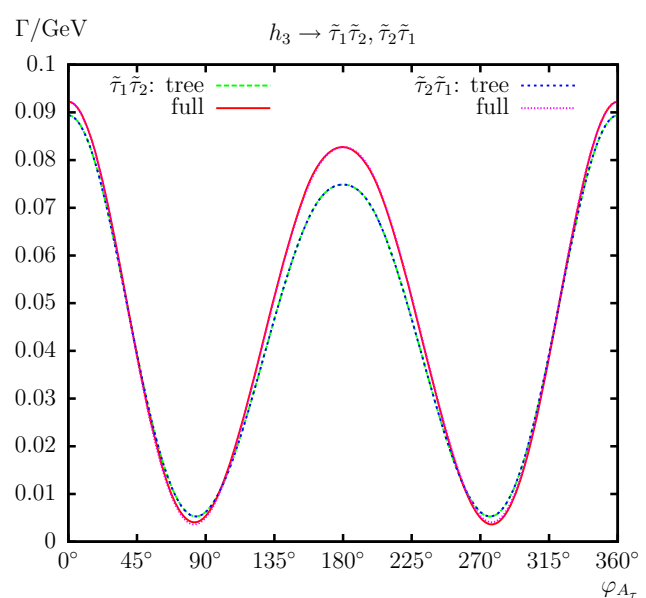

show the complex phase $\varphi_{A_{\tau}}$ varied for $h_{2}$ decays (left) and $h_{3}$ decays (right) with parameters chosen according to $\mathrm{S} 1$ (see Table 2)

decaying Higgs boson. While below MC2 we find $\Gamma\left(h_{2} \rightarrow\right.$ $\left.\tilde{t}_{1} \tilde{t}_{2}, \tilde{t}_{2} \tilde{t}_{1}\right) \gg \Gamma\left(h_{3} \rightarrow \tilde{t}_{1} \tilde{t}_{2}, \tilde{t}_{2} \tilde{t}_{1}\right)$, above MC2 we have correspondingly $\Gamma\left(h_{2} \rightarrow \tilde{t}_{1} \tilde{t}_{2}, \tilde{t}_{2} \tilde{t}_{1}\right) \ll \Gamma\left(h_{3} \rightarrow \tilde{t}_{1} \tilde{t}_{2}, \tilde{t}_{2} \tilde{t}_{1}\right)$, as can be clearly observed in the upper plot of Fig. 9 .

We now turn to the phase dependence of the decay width shown in S2, i.e. for $M_{H^{ \pm}}=1400 \mathrm{GeV}$, where the left (right) plot in Fig. 9 shows the dependence of $\Gamma\left(h_{2} \rightarrow \tilde{t}_{1} \tilde{t}_{2}, \tilde{t}_{2} \tilde{t}_{1}\right)$ $\left(\Gamma\left(h_{3} \rightarrow \tilde{t}_{1} \tilde{t}_{2}, \tilde{t}_{2} \tilde{t}_{1}\right)\right)$ on $\varphi_{A_{t}}$. In the lower left plot one can observe that already the tree-level result (green dashed) and the tree of the conjugated process (blue short dashed) are asymmetric and depend strongly on the phase. The asymmetry at the tree level is due to the contribution from the $\hat{\mathbf{Z}}$ matrix, which is not in general unitary and depends via the stop contributions to the Higgs boson self-energies on $\varphi_{A_{t}}$; see Ref. [33]. While for $\varphi_{A_{t}} \sim 180^{\circ}$ a width of about $1.5 \mathrm{GeV}$ is observed, for $\varphi_{A_{t}} \sim 0^{\circ}$ a three times higher decay width is found. The full corrections for $\varphi_{A_{t}}$ varied are $\lesssim+12 \%$ for S2, while the SQCD corrections overestimate the full corrections up to $30 \%$. In the lower right plot of Fig. 9, where we show the $\varphi_{A_{t}}$ dependence of the $h_{3}$ decay one can see that as 


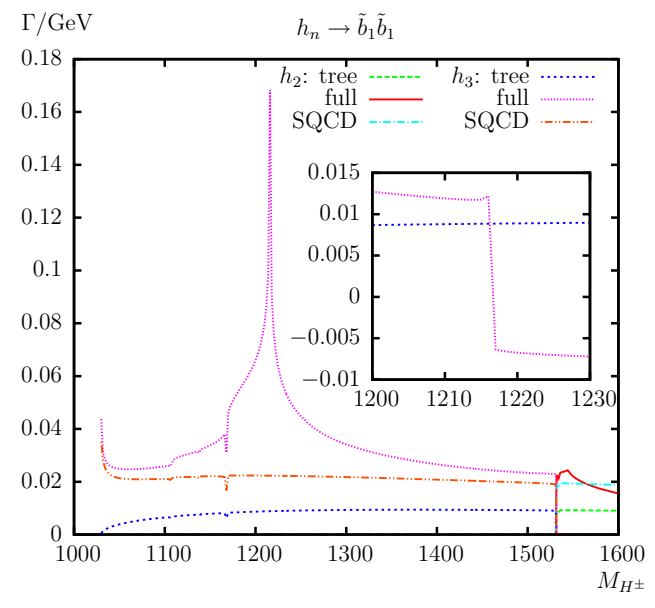

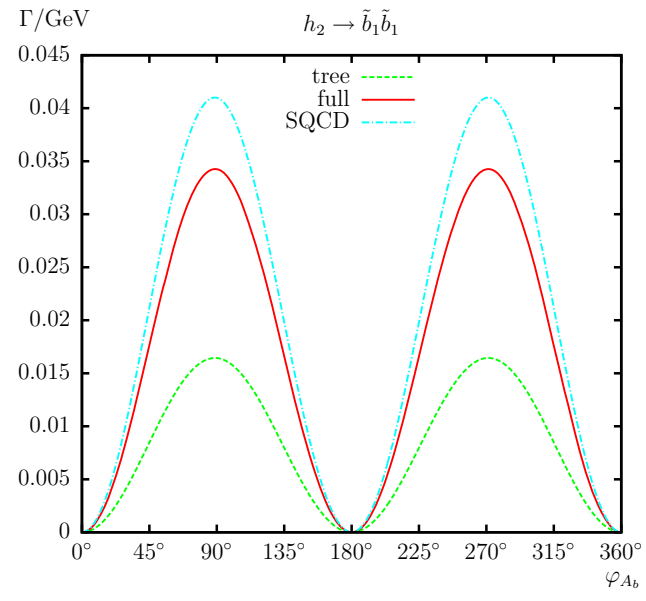

Fig. $12 \Gamma\left(h_{n} \rightarrow \tilde{b}_{1} \tilde{b}_{1}\right)$. Tree-level, full, and SQCD one-loop corrected partial decay widths are shown. The upper plot shows the partial decay width with $M_{H^{ \pm}}$varied; the lower plots

for the $h_{2}$ case already the tree-level results (green dashed) and the tree of the conjugated process (blue short dashed) depend strongly on the phase and exhibit an asymmetry. The latter is again due to the contribution from the $\hat{\mathbf{Z}}$ matrix. The relative corrections for $\varphi_{A_{t}}$ are up to $\sim+29 \%$ for $\mathrm{S} 2$. The SQCD corrections are smaller and would underestimate the full corrections by more than $50 \%$.

In Fig. 10 we present the results for the decays $h_{n} \rightarrow$ $\tilde{b}_{1} \tilde{b}_{2}, \tilde{b}_{2} \tilde{b}_{1}$, where in the upper (lower) row we show the dependence on $M_{H^{ \pm}}\left(\varphi_{A_{b}}\right)$. In the upper row plot the dips/thresholds TN11, TN12, TN15, TN16, and TN17 appear. The step at $M_{H^{ \pm}} \approx 1184 \mathrm{GeV}$ is an anomalous threshold (see p. 376 in Ref. [115]) and could be traced back to the $C$-functions $C_{0,1,2}\left(m_{h_{2}}^{2}, m_{\tilde{b}_{1}}^{2}, m_{\tilde{b}_{2}}^{2}, m_{\tilde{t}_{i}}^{2}, m_{\tilde{t}_{j}}^{2}, M_{W}^{2}\right)$ with $i \neq j$. The parameter regions very close to these steps are expected to be somewhat unreliable and should be interpreted with care. At $M_{H^{ \pm}}=1400 \mathrm{GeV}$ the full one-loop corrections to the $h_{2}$ decay reach only $\sim+6 \%$, while the SQCD corrections would overestimate this by a factor of $\sim 2.5$. Now we turn to the corresponding $h_{3}$ decay. The first three dips and the

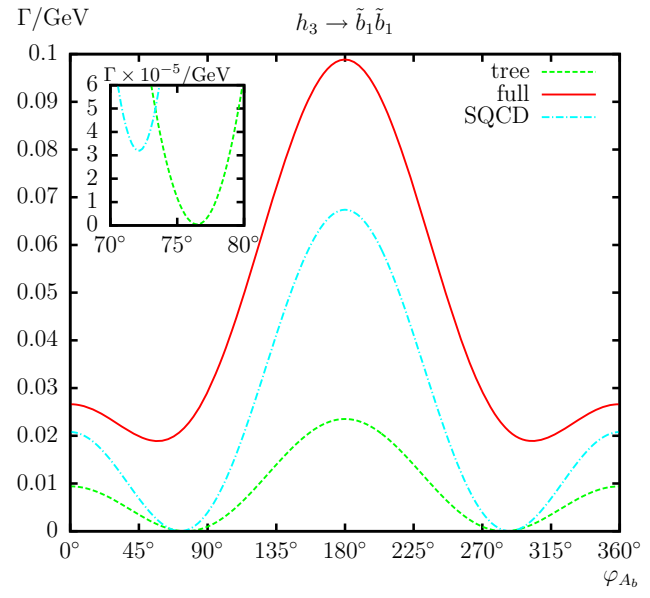

show the complex phase $\varphi_{A_{b}}$ varied for $h_{2}$ decays (left) and $h_{3}$ decays (right) with parameters chosen according to S2 (see Table 2)

step at $M_{H^{ \pm}} \approx 1182 \mathrm{GeV}$ are the same as for the $h_{2}$ decay; see above. For the decay of the $h_{3}$ at $M_{H^{ \pm}}=1400 \mathrm{GeV}$ we find full corrections at the level of $+17 \%$, where the SQCD results are only slightly larger. As in Fig. 9 one can observe the MC2 with an "interchange" of $h_{2}$ and $h_{3}$.

In the lower left plot of Fig. 10 we present $\Gamma\left(h_{2} \rightarrow\right.$ $\left.\tilde{b}_{1} \tilde{b}_{2}, \tilde{b}_{2} \tilde{b}_{1}\right)$ as a function of $\varphi_{A_{b}}$ in $\mathrm{S} 2$. The variation with $\varphi_{A_{b}}$ is found to be very large, full relative corrections are up to $\sim+90 \%$ for S2, where the SQCD corrections account for about $60 \%$ of those. This can partially be attributed to the very small tree level within the region $60^{\circ} \lesssim \varphi_{A_{b}} \lesssim 300^{\circ}$. Furthermore, a very strong asymmetry between one decay and its complex conjugate can be observed, reaching up to $60 \%$. In the lower right plot the corresponding results for the $h_{3}$ decay are shown. One can see that again already the tree-level result (green dashed) and the tree of the conjugated process (blue short dashed) are asymmetric, which is caused again by the $\hat{\mathbf{Z}}$ matrix contribution, where $\varphi_{A_{b}}$ enters via the $\tilde{b}$ contributions to the Higgs-boson self-energies. As in the $h_{2}$ case the size of the corrections shows also a large variation 


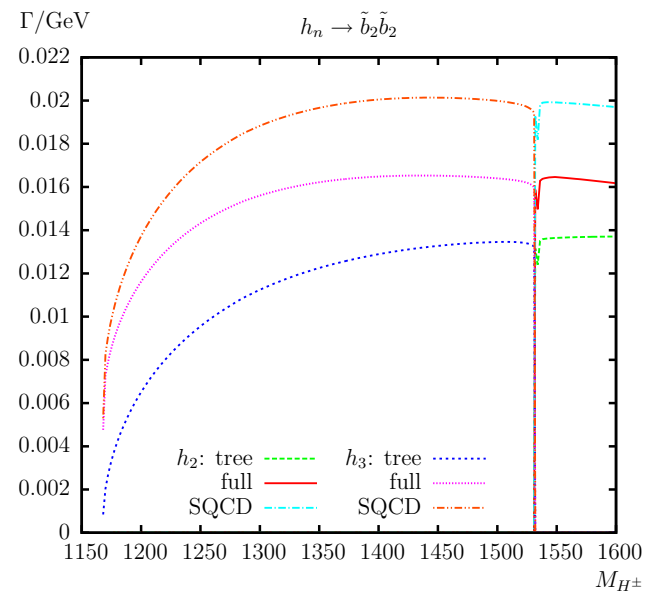

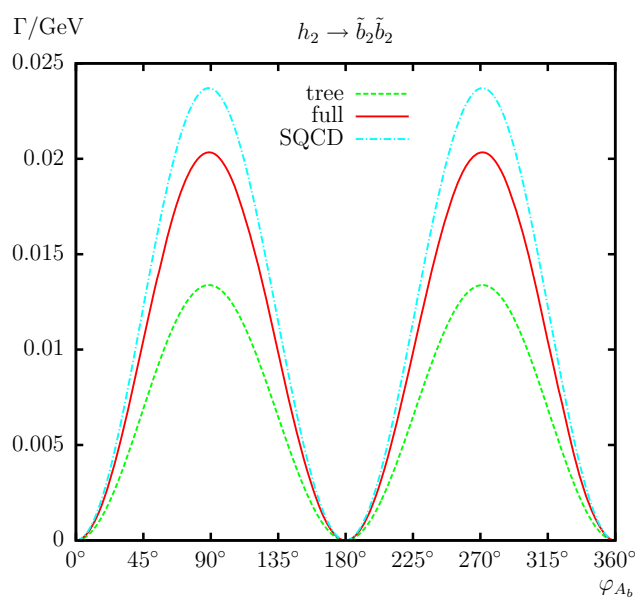

Fig. $13 \Gamma\left(h_{n} \rightarrow \tilde{b}_{2} \tilde{b}_{2}\right)$. Tree-level, full, and SQCD one-loop corrected partial decay widths are shown. The upper plot shows the partial decay width with $M_{H^{ \pm}}$varied; the lower plots

with $\varphi_{A_{b}}$. The full relative corrections are up to $\sim+66 \%$ for S2, where the SQCD corrections are only slightly smaller.

The third "mixed case", the decays $h_{n} \rightarrow \tilde{\tau}_{1} \tilde{\tau}_{2}, \tilde{\tau}_{2} \tilde{\tau}_{1}$, is shown in Fig. 11. As before, the upper plots depict the result as a function of $M_{H^{ \pm}}$, whereas the lower row presents the $\varphi_{A_{\tau}}$ dependence. Here it should be noted that in many MSSM scenarios (as in the numerical example chosen here) the scalar taus are lighter than the scalar quarks. Consequently, even a small decay width could result in a relatively large BR. We start our numerical description with the $h_{2}$ decay as a function of $M_{H^{ \pm}}$. The first dip is the threshold TN5; see Table 4. The second (small) dip is the threshold TN9. ${ }^{14}$ Furthermore, the dips/thresholds TN11, TN12, TN15, TN16, and TN17 appear. At $M_{H^{ \pm}}=1000 \mathrm{GeV}$ the full one-loop corrections

\footnotetext{
$\overline{14}$ It should be noted that the "squark" thresholds (in a $h_{n}$ decay into sleptons) enter into the tree level only via the $\hat{\mathbf{Z}}$ matrix contribution. Via $2 \operatorname{Re}\left\{\mathcal{M}_{\text {tree }}^{*} \mathcal{M}_{1-\text { loop }}\right\}$ these effects propagate also into the loop corrections. (Of course there are in addition pure loop corrections from the squark-squark-slepton-slepton couplings; see third row, third column of Fig. 1.)
}

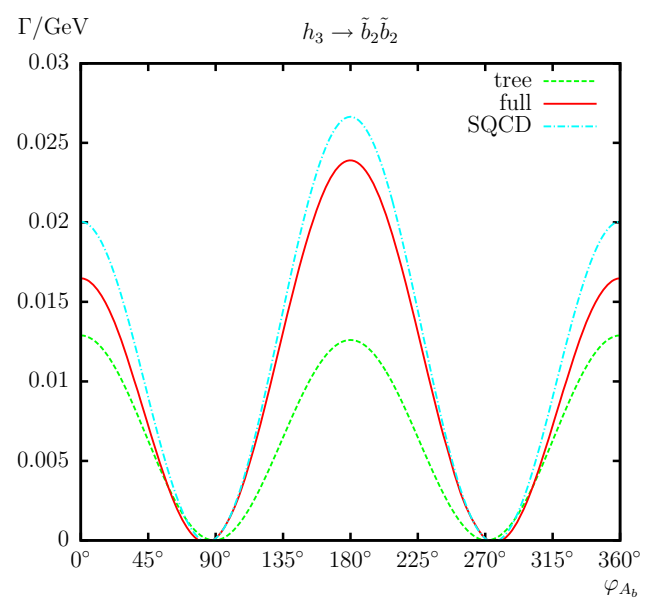

show the complex phase $\varphi_{A_{b}}$ varied for $h_{2}$ decays (left) and $h_{3}$ decays (right) with parameters chosen according to S2 (see Table 2)

reach $\sim-9 \%$. In the same plot we show also the results for the $h_{3}$ decay. Here the dips/thresholds TN3, TN6, TN7, TN10, TN13, and TN14 appear. At $M_{H^{ \pm}}=1000 \mathrm{GeV}$ the full one-loop corrections reach $\sim+3 \%$. The two mass crossings, $\mathrm{MC} 1$ and $\mathrm{MC} 2$, can again be observed, where $h_{2}$ and $h_{3}$ interchange their $\mathcal{C P}$ character.

We now turn to the results for the $h_{2}\left(h_{3}\right)$ decay as a function of $\varphi_{A_{\tau}}$ in the lower left (right) plot of Fig. 11. For the $h_{2}$ decay the relative corrections for $\varphi_{A_{\tau}}=80^{\circ}, 180^{\circ}, 280^{\circ}$ are up to $\sim+7 \%$ in S1. For the $h_{3}$ decay, on the other hand, the relative corrections for $\varphi_{A_{\tau}}=82^{\circ}, 180^{\circ}$ are up to $\sim-24 \%, \sim+10 \%$ in S1. The asymmetry is too small to be visible in the plot.

Next we consider $h_{n}$ decays into sfermions with equal sfermion indices and it should be noted that the $A \tilde{f}_{i} \tilde{f}_{i}$ $(i=1,2)$ couplings are exactly zero in case of real input parameters.

In Fig. 12 we present the results for the decays $h_{n} \rightarrow \tilde{b}_{1} \tilde{b}_{1}$. The dependence on $M_{H^{ \pm}}$is shown in the upper plot, whereas the dependence on $\varphi_{A_{b}}$ for $M_{H^{ \pm}}=1400 \mathrm{GeV}$ is given in the 

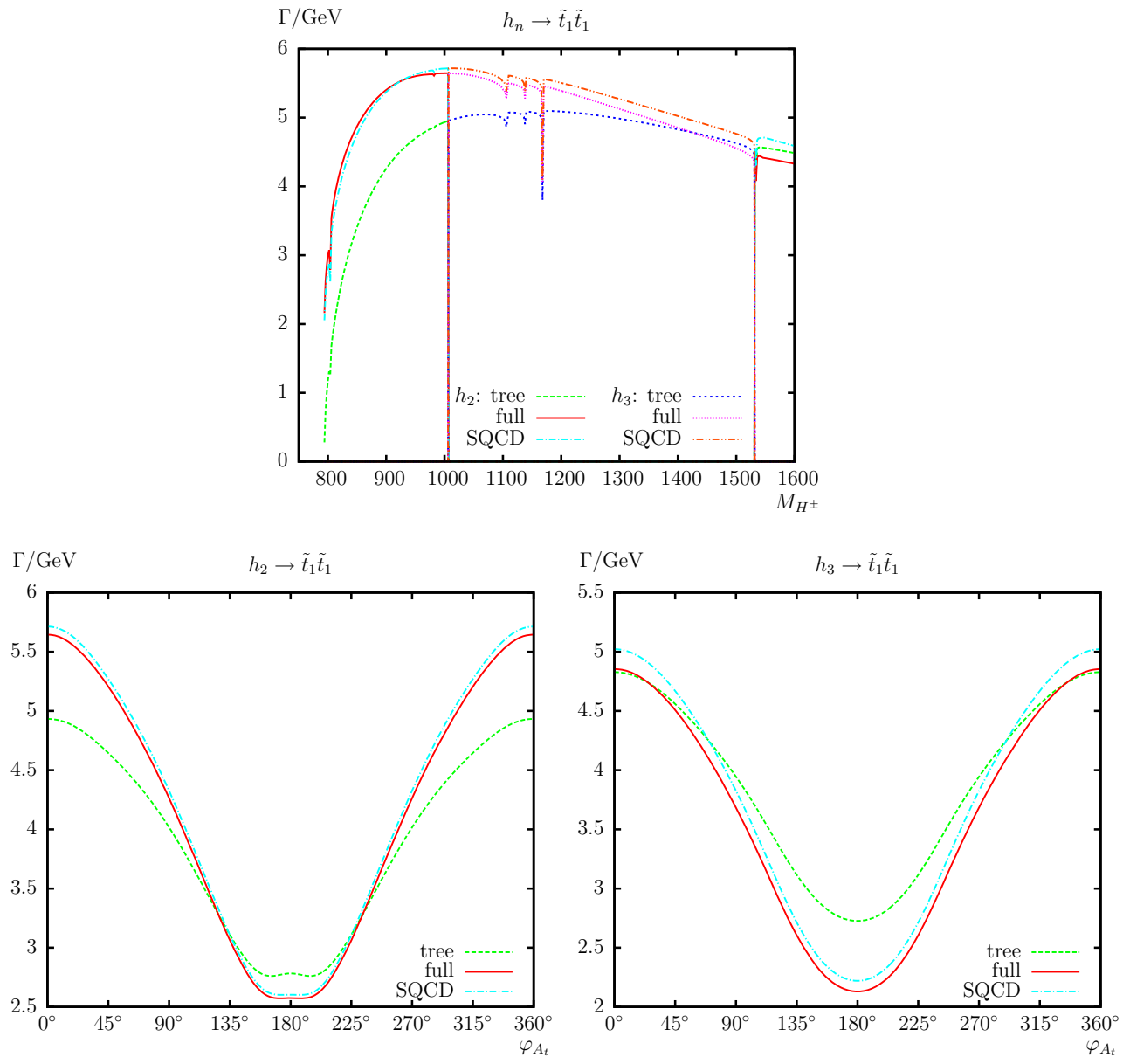

Fig. $14 \Gamma\left(h_{n} \rightarrow \tilde{t}_{1} \tilde{t}_{1}\right)$. Tree-level, full, and SQCD one-loop corrected partial decay widths are shown. The upper plot shows the partial decay width with $M_{H^{ \pm}}$varied; the lower plots show the complex phase $\varphi_{A_{b}}$ varied for $h_{2}$ decays (left, S1) and $h_{3}$ decays (right, S2)

lower plots. We start with $\Gamma\left(h_{2} \rightarrow \tilde{b}_{1} \tilde{b}_{1}\right)$ in the upper plot. Only above MC2 this decay width becomes non-zero. The peak at $M_{H^{ \pm}} \approx 1545 \mathrm{GeV}$ (red line) is the threshold TN18. Furthermore, the tree-level decay width $\Gamma\left(h_{2} \rightarrow \tilde{b}_{1} \tilde{b}_{1}\right)$ is accidentally very small for the parameter set chosen; see Table 2. Because of this smallness, the relative size of the one-loop correction becomes larger than the tree level, and it can even turn negative. Therefore, in this case (i.e. negative decay width) we added $\left|\mathcal{M}_{1 \text {-loop }}\right|^{2}$ to the full one-loop result to obtain a positive decay width. The full relative corrections are $\sim+73 \%$ at $M_{H^{ \pm}}=1600 \mathrm{GeV}$ and the SQCD corrections are $\sim+80 \%$. Also shown in this plot is the decay $h_{3} \rightarrow \tilde{b}_{1} \tilde{b}_{1}$, which is non-zero below MC2. For this decay the dips/thresholds TN10, TN13, and TN14 appear. The large "spike" at $M_{H^{ \pm}} \approx 1216 \mathrm{GeV}$ is caused by the addition of the two-loop contribution $\left|\mathcal{M}_{1-\text { loop }}\right|^{2}$ as explained above (formally it is caused by the anomalous thresholds of the $C$ functions $C_{0,1,2}\left(m_{h_{3}}^{2}, m_{\tilde{b}_{1}}^{2}, m_{\tilde{b}_{1}}^{2}, m_{\tilde{t}_{i}}^{2}, m_{\tilde{t}_{j}}^{2}, M_{W}^{2}\right)$ with $\left.i \neq j\right)$. Without the two-loop contribution it appears as step (see the inlay in the upper plot of Fig. 12) similar to Fig. 10. Because of the smallness of the tree, the full relative corrections reach $\sim+183 \%$ at $M_{H^{ \pm}}=1400 \mathrm{GeV}$. Here the SQCD corrections are smaller with $\sim+92 \%$.

In the lower left plot of Fig. 12 we show the $h_{2}$ decay with the complex phase $\varphi_{A_{b}}$ varied at $M_{H^{ \pm}}=1400 \mathrm{GeV}$. For $\varphi_{A_{b}}=0^{\circ}, 180^{\circ}, 360^{\circ}$, i.e. real parameters the $h_{2}$ decay is purely $\mathcal{C P}$-odd, and thus the decay width is zero. For complex values of the phase small, but non-zero values are reached. Here, for the same reasons as in the upper plot the loop corrections can be larger than the level and reach actually $\sim+108 \%$ (and $\sim+125 \%$ for SQCD) at $\varphi_{A_{b}}=90^{\circ}, 270^{\circ}$. In the lower right plot of Fig. 12 we show the $h_{3}$ decay with the complex phase $\varphi_{A_{b}}$ varied at $M_{H^{ \pm}}=1400 \mathrm{GeV}$. Here (for the same reasons as in the upper plot) the loop corrections can be larger then the tree level (and for consistency with the upper plot we also added $\left|\mathcal{M}_{\text {1-loop }}\right|^{2}$ here) and reach up to $\sim+320 \%$ (and $\sim+165 \%$ for SQCD) at $\varphi_{A_{b}}=180^{\circ}$. It should be noted that the SQCD decay width at $\varphi_{A_{b}} \approx 76^{\circ}, 284^{\circ}$ does not go to zero due to $\left|\mathcal{M}_{1 \text {-loop }}\right|^{2}$, but just reaches a (very) small value of $\Gamma\left(h_{3} \rightarrow \tilde{b}_{1} \tilde{b}_{1}\right)$ (see the inlay in the lower right plot of Fig. 12). For S2 we have $h_{3} \sim H$ and the $\hat{\mathbf{Z}}$ matrix depends rather weak on $A_{b}$, i.e. 


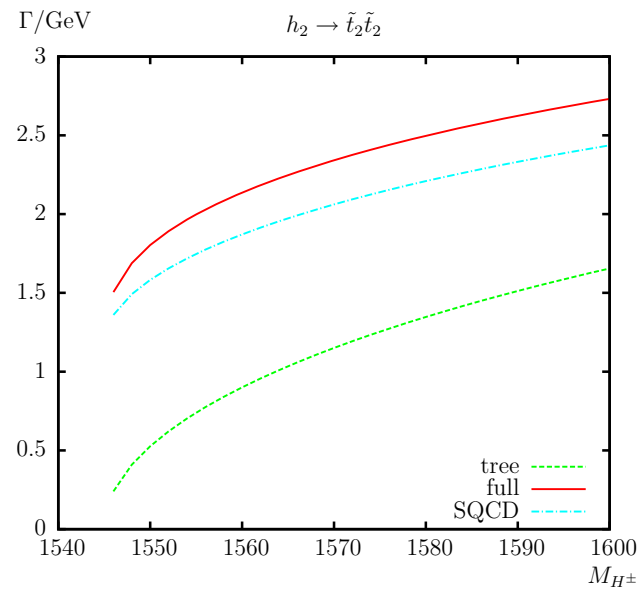

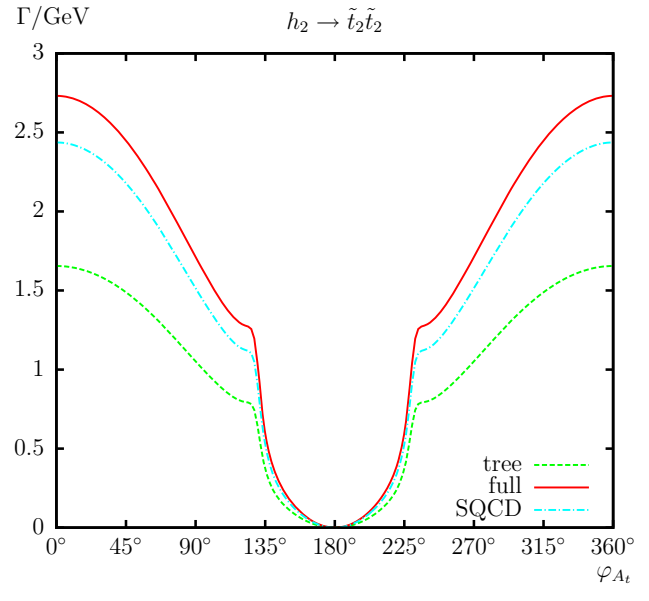

Fig. $15 \Gamma\left(h_{n} \rightarrow \tilde{t}_{2} \tilde{t}_{2}\right)$. Tree-level, full, and SQCD one-loop corrected partial decay widths are shown. The upper plot shows the partial decay width with $M_{H^{ \pm}}$varied; the lower plots

the main effect on the decay width stems continuously from the $\hat{\mathbf{Z}}$ matrix element $\hat{Z}_{32}$. In contrast to the left plot the tree decay width is zero at $\varphi_{A_{b}} \approx 76^{\circ}, 284^{\circ}$, because the relevant $\left(\propto \hat{Z}_{32}\right)$ real part of the coupling $C\left(h_{3}, \tilde{b}_{1}, \tilde{b}_{1}\right)$, see Ref. [87], accidentally goes through zero.

In Fig. 13 we present the decay $h_{n} \rightarrow \tilde{b}_{2} \tilde{b}_{2}$, in full analogy to Fig. 12. The same behavior of $h_{2}$ and $h_{3}$ concerning MC2 can be observed. The full relative corrections for the $h_{2}$ decay are $\sim+18 \%$ at $M_{H^{ \pm}}=1600 \mathrm{GeV}$, where the pure SQCD corrections would overestimate this correction by a factor $\sim 1.8$. The decay $h_{3} \rightarrow \tilde{b}_{2} \tilde{b}_{2}$ is again non-zero only below MC2. The dip (hardly visible at $M_{H^{ \pm}} \approx 1168 \mathrm{GeV}$ ) in the $h_{3}$ decay is the threshold TN14. The full relative corrections are $\sim+28 \%$ at $M_{H^{ \pm}}=1400 \mathrm{GeV}$ and the SQCD corrections are larger by about a factor of 1.6.

In the lower left plot of Fig. 13 we show the variation of $\Gamma\left(h_{2} \rightarrow \tilde{b}_{2} \tilde{b}_{2}\right)$ with $\varphi_{A_{b}}$ at $M_{H^{ \pm}}=1400 \mathrm{GeV}$. Here the loop corrections reach $\sim+52 \%$ (and $\sim+60 \%$ for SQCD) at $\varphi_{A_{b}}=90^{\circ}, 270^{\circ}$. The decay width goes to zero for real $A_{b}$ due to the $\mathcal{C P}$-nature of the $h_{2}$. In the lower right plot of Fig. 13 we show $\Gamma\left(h_{3} \rightarrow \tilde{b}_{2} \tilde{b}_{2}\right)$ with $\varphi_{A_{b}}$ var-

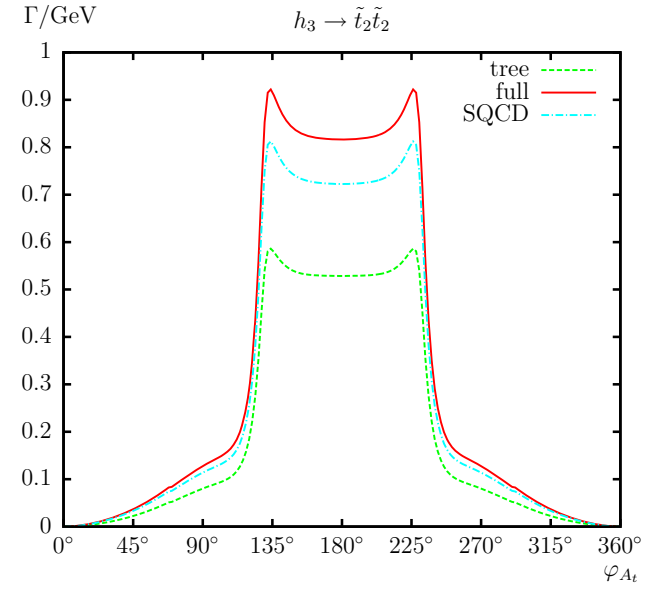

show the complex phase $\varphi_{A_{t}}$ varied for $h_{2}$ decays (left) and $h_{3}$ decays (right) with parameters chosen according to S3 (see Table 2)

ied at $M_{H^{ \pm}}=1400 \mathrm{GeV}$. Here the loop corrections reach $\sim+90 \%$ at $\varphi_{A_{b}}=180^{\circ}$ and are slightly overestimated in the pure SQCD case. The tree decay width goes to zero for $\varphi_{A_{b}} \approx 85^{\circ}, 275^{\circ}$, for the same reasons as discussed above for Fig. 12. We have again $h_{3} \sim H$, i.e. the main effect on the decay width stems from $\hat{Z}_{32}$.

We now turn to the neutral Higgs decay to scalar top quarks, which are shown in full analogy to the decay to scalar bottom quarks above. In Fig. 14 we present the decay $h_{n} \rightarrow \tilde{t}_{1} \tilde{t}_{1}$. In the upper row we show the results as a function of $M_{H^{ \pm}}$. The dips/thresholds appearing in the $h_{2}$ decay are TN5 and TN8. The full relative corrections are $\sim+14 \%$ at $M_{H^{ \pm}}=1000 \mathrm{GeV}$ (i.e. S1) and the SQCD corrections are $\sim+16 \%$. The decay width turns zero between $\mathrm{MC} 1$ and MC2 and reaches non-zero values below MC1 and above MC2. Reversely, we find non-zero values for $\Gamma\left(h_{3} \rightarrow \tilde{t}_{1} \tilde{t}_{1}\right)$ only between $\mathrm{MC} 1$ and $\mathrm{MC} 2$. The dips in the $h_{3}$ decay are the thresholds TN10, TN13, and TN14. The full relative corrections at $M_{H^{ \pm}}=1400 \mathrm{GeV}$ are accidentally small and reach only $+1 \%$, where the pure SQCD corrections reach $\sim+4 \%$. 

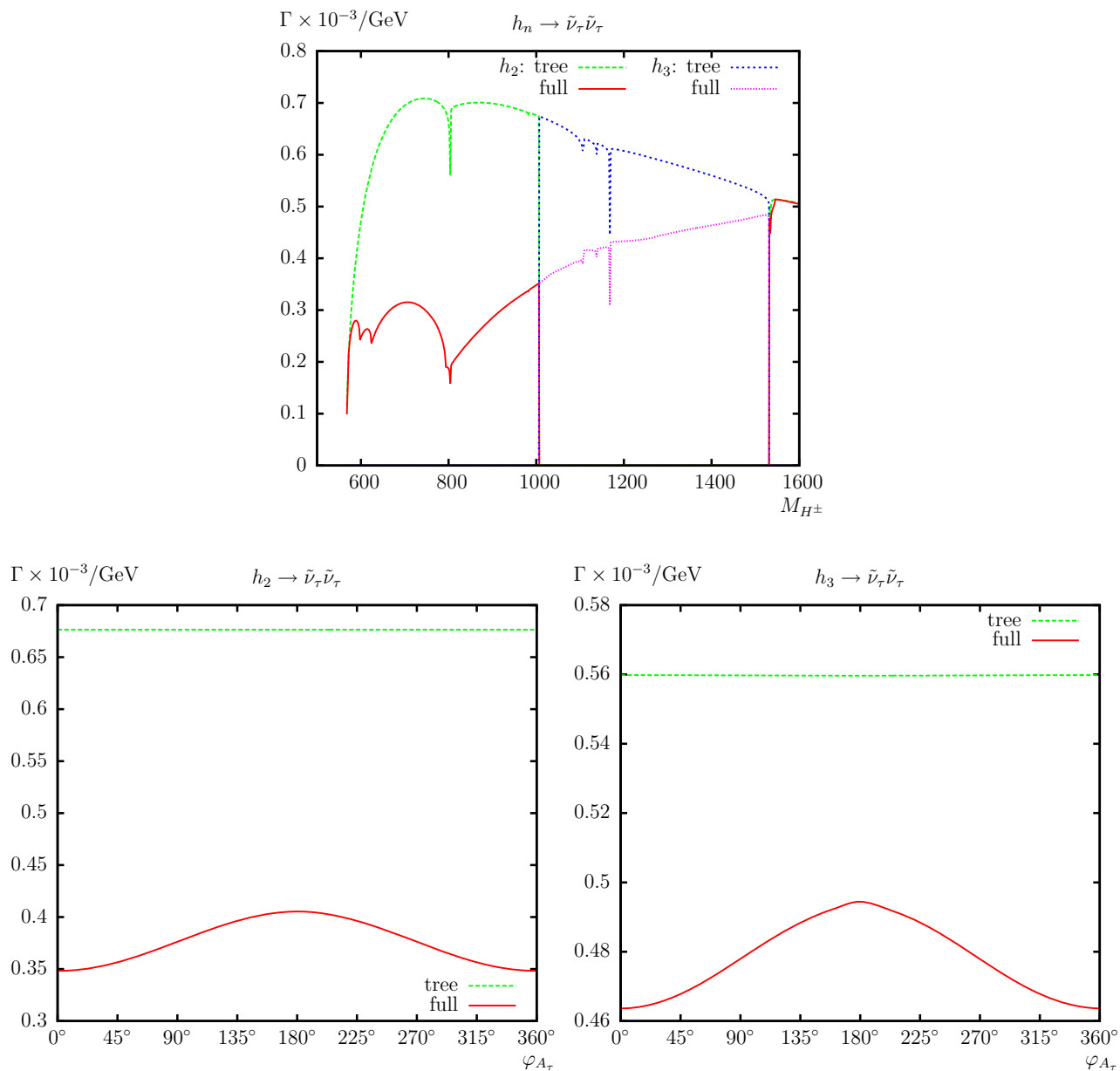

Fig. $16 \Gamma\left(h_{n} \rightarrow \tilde{v}_{\tau} \tilde{v}_{\tau}\right)$. Tree-level and full one-loop corrected partial decay widths are shown. The upper plot shows the partial decay width with $M_{H^{ \pm}}$varied; the lower plots show the complex phase $\varphi_{A_{\tau}}$ varied for $h_{2}$ decays (left, S1) and $h_{3}$ decays (right, S2)

In the lower left plot of Fig. 14 we show the $h_{2}$ decay with the complex phase $\varphi_{A_{t}}$ varied at $M_{H^{ \pm}}=1000 \mathrm{GeV}$. Here the loop corrections can vary between $\sim+14 \%$ for $\varphi_{A_{t}} \sim 0^{\circ}, 360^{\circ}$ and $\sim-7 \%$ at $\varphi_{A_{t}}=180^{\circ}$, where the SQCD corrections in this case are a good approximation to the full result. In the lower right plot of Fig. 14 we show the $h_{3}$ decay with $\varphi_{A_{t}}$ varied at $M_{H^{ \pm}}=1400 \mathrm{GeV}$. Here the loop corrections are close to zero for real positive values of $A_{b}$, where the EW corrections compensate the SQCD contributions. The full corrections can reach $\sim-22 \%$ and $\sim-19 \%$ for SQCD) at $\varphi_{A_{t}}=180^{\circ}$.

The final decays involving stops are shown in Fig. 15. The results as a function of $M_{H^{ \pm}}$are given in the upper plot. Due to the large values of $m_{\tilde{t}_{2}}$ for real parameters only $\Gamma\left(h_{2} \rightarrow \tilde{t}_{2} \tilde{t}_{2}\right)$ reaches non-zero values, because $h_{2} \sim H$ and $h_{3} \sim A$. The full relative corrections for the $h_{2}$ decay are $\sim+65 \%$ at $M_{H^{ \pm}}=1600 \mathrm{GeV}$ (i.e. S3) and the SQCD corrections reach $\sim+47 \%$.

In the lower left plot of Fig. 15 we show $\Gamma\left(h_{2} \rightarrow \tilde{t}_{2} \tilde{t}_{2}\right)$ with the complex phase $\varphi_{A_{t}}$ varied at $M_{H^{ \pm}}=1600 \mathrm{GeV}$. The smooth structure around $\varphi_{A_{t}}=130^{\circ}, 230^{\circ}$ is not a threshold but an interplay of $\hat{Z}_{22}$ multiplied by the corresponding (real and imaginary) part of the coupling $C\left(h_{2}, \tilde{t}_{2}, \tilde{t}_{2}\right)$; see Ref. [87]. For $\varphi_{A_{t}}=180^{\circ}$ the decay width goes to zero, since there is an evolving mass crossing from $\varphi_{A_{t}}=0^{\circ}\left(h_{2} \sim H\right)$ to $\varphi_{A_{t}}=180^{\circ}\left(h_{2} \sim A\right)$ due to the strong dependence of the $\hat{\mathbf{Z}}$ matrix from $A_{t}$, i.e. a strong variation with $\varphi_{A_{t}}$. The loop corrections can reach $\sim+63 \%$ (and $\sim+44 \%$ for SQCD) at $\varphi_{A_{t}}=90^{\circ}, 270^{\circ}$. The $h_{3}$ decay is non-zero above MC2 only for complex parameters. In the lower right plot of Fig. 15 we show $\Gamma\left(h_{3} \rightarrow \tilde{t}_{2} \tilde{t}_{2}\right)$ with $\varphi_{A_{t}}$ varied at $M_{H^{ \pm}}=1600 \mathrm{GeV}$. The smooth structure around $\varphi_{A_{t}}=135^{\circ}, 225^{\circ}$ is mainly a numerical effect of the $\hat{\mathbf{Z}}$ matrix element $\hat{Z}_{33}$. The loop corrections can reach $\sim+54 \%$ (and $\sim+37 \%$ for SQCD) at $\varphi_{A_{t}}=180^{\circ}$.

We finish our numerical analysis with the remaining decays to scalar leptons. In Fig. 16 the decay widths for $h_{n} \rightarrow \tilde{v}_{\tau} \tilde{v}_{\tau}$ are shown. In the upper plot the results as a function of $M_{H^{ \pm}}$are given. The decay $h_{2} \rightarrow \tilde{v}_{\tau} \tilde{\nu}_{\tau}$ for real parameters is non-zero below MC1 and above MC2 due to the $\mathcal{C P}$-structure of $h_{2}$. The dips/thresholds appearing for the $h_{2}$ decay are TN1, TN2, TN4, TN5, and TN8. The full relative 

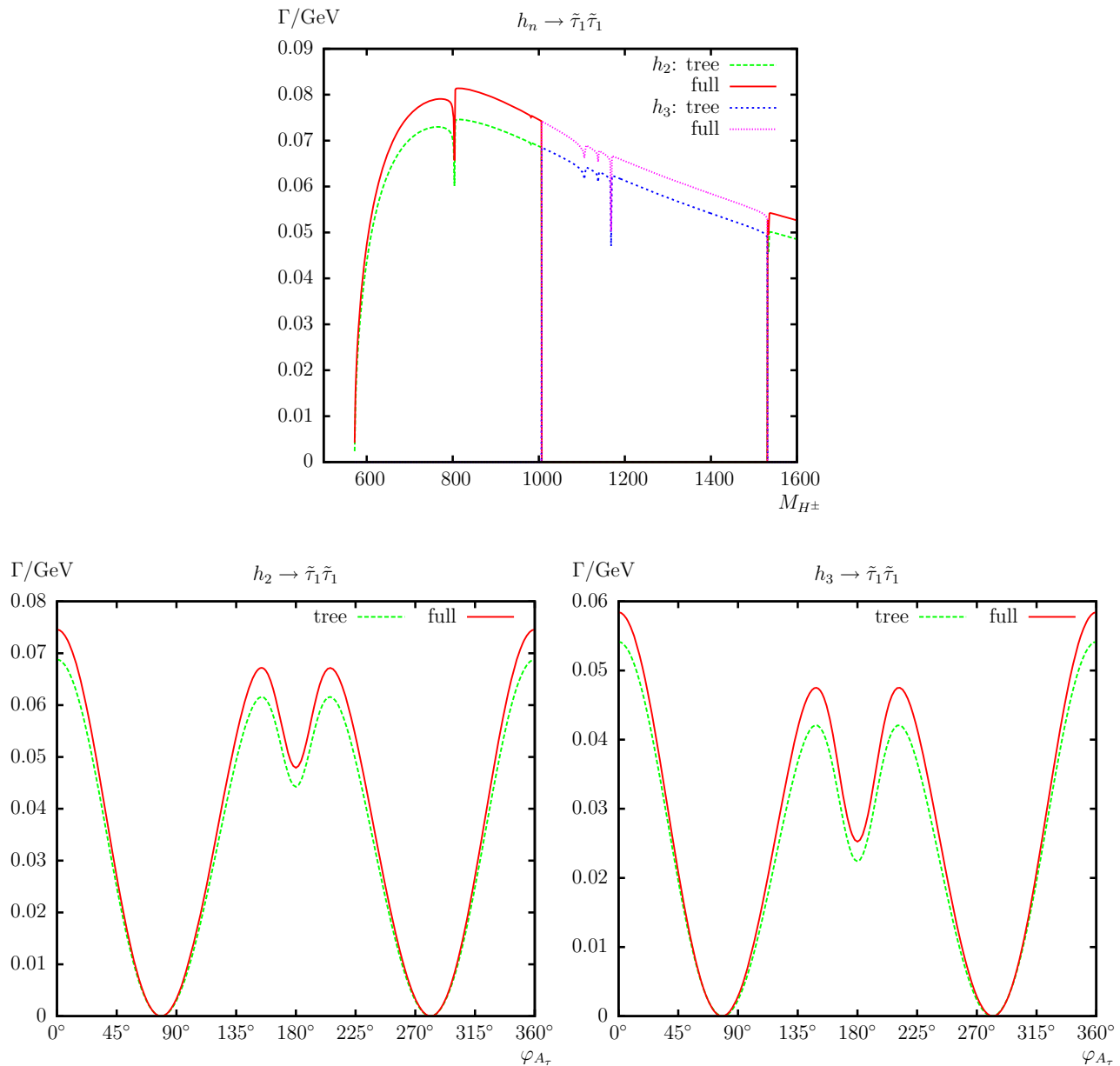

Fig. $17 \Gamma\left(h_{n} \rightarrow \tilde{\tau}_{1} \tilde{\tau}_{1}\right)$. Tree-level and full one-loop corrected partial decay widths are shown. The upper plot shows the partial decay width with $M_{H^{ \pm}}$varied; the lower plots show the complex phase $\varphi_{A_{\tau}}$ varied for $h_{2}$ decays (left, S1) and $h_{3}$ decays (right, S2)

corrections are found to be $\sim-48 \%$ at $M_{H^{ \pm}}=1000 \mathrm{GeV}$ (i.e. S1). Correspondingly, the decay $h_{3} \rightarrow \tilde{v}_{\tau} \tilde{\nu}_{\tau}$ for real parameters is non-zero only between MC1 and MC2. The dips are the thresholds TN10, TN13, and TN14. The full relative corrections are $\sim-17 \%$ at $M_{H^{ \pm}}=1400 \mathrm{GeV}$ (i.e. S2).

The dependence on $\varphi_{A_{\tau}}$ is shown for the $h_{2}\left(h_{3}\right)$ decay in the lower left (right) plot of Fig. 16 for $M_{H^{ \pm}}=$ 1000 (1400) GeV. The tree decay width is independent from $\varphi_{A_{\tau}}$ due to the absence of $A_{\tau}$ in the corresponding couplings $C\left(h_{n}, \tilde{v}_{\tau}, \tilde{v}_{\tau}\right)$, see Ref. [87], which can be clearly observed in both lower plots. For the $h_{2}$ decay the loop corrections can reach $\sim-40 \%$ at $\varphi_{A_{\tau}}=180^{\circ}$. For the $h_{3}$ decay they can reach $\sim-12 \%$ at $\varphi_{A_{\tau}}=180^{\circ}$.

In Fig. 17 we present the results for the decays $h_{n} \rightarrow \tilde{\tau}_{1} \tilde{\tau}_{1}$. The upper row shows the decay widths as a function of $M_{H^{ \pm}}$. As before, the decay width of $h_{2}$ is non-zero for real parameters only below MC1 and above MC2, whereas the $h_{3}$ decay width is non-zero between the two mass crossing points. Starting with $h_{2}$, the dips/thresholds are TN5 and TN8. The full relative corrections are $\sim+8 \%$ at $M_{H^{ \pm}}=1000 \mathrm{GeV}$ (i.e. S1). The dips of the $h_{3}$ decay are (again) the thresh- olds TN10, TN13 and TN14. The full relative corrections at $M_{H^{ \pm}}=1400 \mathrm{GeV}$ (i.e. S2) are $\sim+8 \%$.

In the lower left plot of Fig. 17 we show the $h_{2}$ decay with $\varphi_{A_{\tau}}$ varied at $M_{H^{ \pm}}=1000 \mathrm{GeV}$. Here the loop corrections can reach $\sim+9 \%$ around $\varphi_{A_{\tau}} \sim 140^{\circ}, 220^{\circ}$. For S1 we have $h_{2} \sim H$ and the $\hat{\mathbf{Z}}$ matrix depends rather weak on $A_{\tau}$, i.e. the main effect on the decay width stems continuously from the $\hat{\mathbf{Z}}$ matrix element $\hat{Z}_{22}$. The tree decay width goes to zero at $\varphi_{A_{b}} \approx 78^{\circ}, 282^{\circ}$, because the relevant $\left(\propto \hat{Z}_{22}\right)$ real part of the coupling $C\left(h_{2}, \tilde{\tau}_{1}, \tilde{\tau}_{1}\right)$, see Ref. [87], accidentally goes through zero. In the lower right plot of Fig. 17 we show $h_{3}$ results as a function of $\varphi_{A_{\tau}}$ with $M_{H^{ \pm}}=1400 \mathrm{GeV}$. Here the loop corrections can reach $\sim+13 \%$ at $\varphi_{A_{\tau}} \sim 140^{\circ}, 220^{\circ}$, and we have $h_{2} \sim H$ in S2, which leads to a vanishing width around $\varphi_{A_{\tau}} \sim 78^{\circ}, 282^{\circ}$. Here, this is again because the relevant $\left(\propto \hat{Z}_{32}\right)$ real part of the coupling $C\left(h_{3}, \tilde{\tau}_{1}, \tilde{\tau}_{1}\right)$ accidentally goes through zero.

Finally, in Fig. 18 we present the results for $\Gamma\left(h_{n} \rightarrow\right.$ $\left.\tilde{\tau}_{2} \tilde{\tau}_{2}\right)$, which are shown in full analogy to $\Gamma\left(h_{n} \rightarrow \tilde{\tau}_{1} \tilde{\tau}_{1}\right)$ above. As before, for real parameters, the $h_{2}$ decay width is found non-zero only below $\mathrm{MC} 1$ and above $\mathrm{MC} 2$, while the 

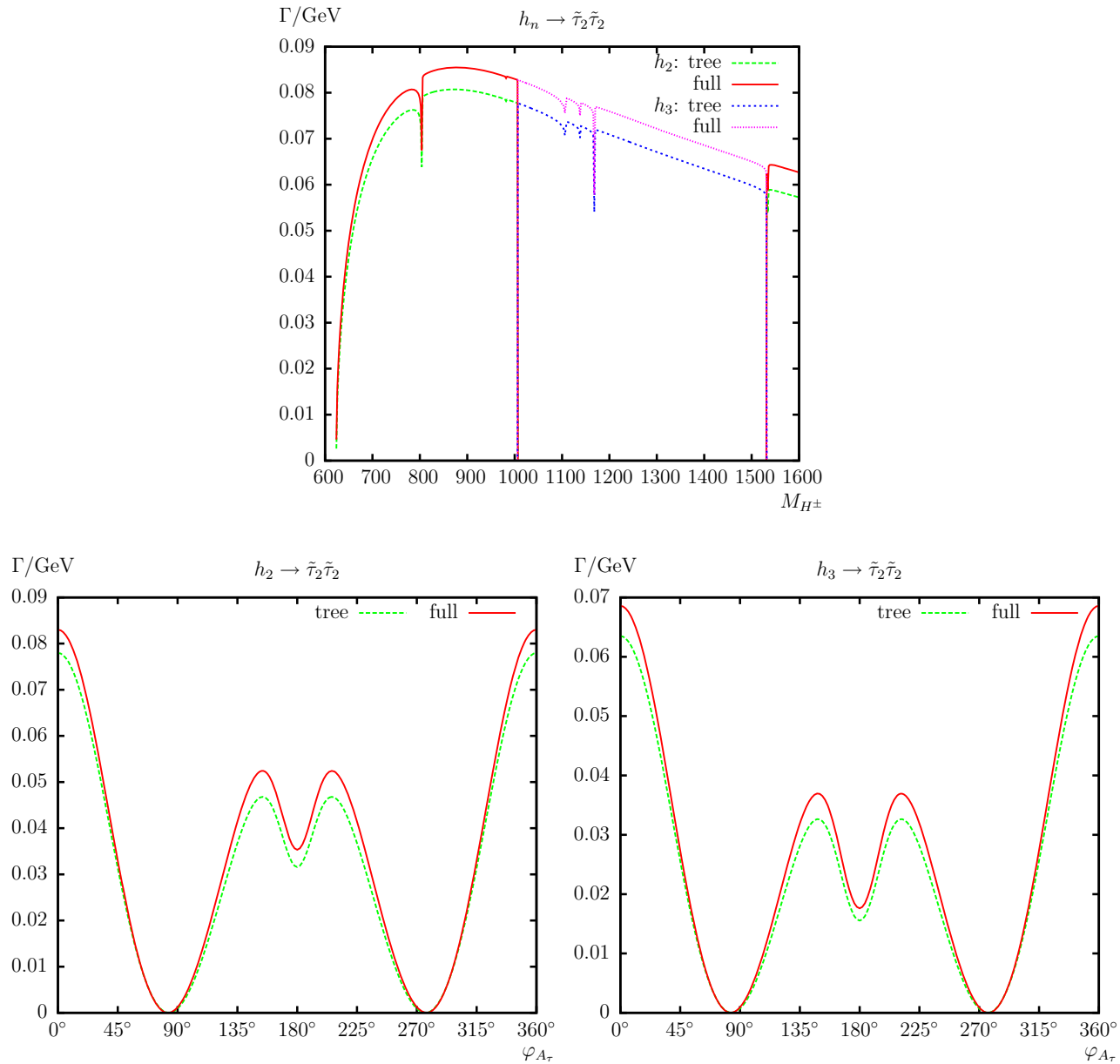

Fig. $18 \Gamma\left(h_{n} \rightarrow \tilde{\tau}_{2} \tilde{\tau}_{2}\right)$. Tree-level and full one-loop corrected partial decay widths are shown. The upper plot shows the partial decay width with $M_{H^{ \pm}}$varied; the lower plots show the complex phase $\varphi_{A_{\tau}}$ varied for $h_{2}$ decays (left, S1) and $h_{3}$ decays (right, S2)

$h_{3}$ width is non-zero only between MC1 and MC2. The $h_{n}$ decay widths as a function of $M_{H^{ \pm}}$exhibit the same dips as in Fig. 17; see above. The full relative corrections to the $h_{2}$ width are $\sim+6 \%$ at $M_{H^{ \pm}}=1000 \mathrm{GeV}$ (i.e. S1). The full relative corrections to the $h_{3}$ decay at $M_{H^{ \pm}}=1400 \mathrm{GeV}$ (i.e. S2) are $\sim+8 \%$.

In the lower left plot of Fig. 18 we show the $h_{2}$ decay with $\varphi_{A_{\tau}}$ varied at $M_{H^{ \pm}}=1000 \mathrm{GeV}$. Here the loop corrections can reach $\sim+12 \%$ at $\varphi_{A_{\tau}} \sim 140^{\circ}, 180^{\circ}, 220^{\circ}$. The decay width goes to zero in analogy to Fig. 17. In the right plot of Fig. 18 the corresponding $h_{3}$ results are shown for $M_{H^{ \pm}}=$ $1400 \mathrm{GeV}$, where we find the same level of higher-order corrections, and the dominating effect of the $\hat{\mathbf{Z}}$ matrix (in combination with the $C\left(h_{2,3}, \tilde{\tau}_{2}, \tilde{\tau}_{2}\right)$ couplings $)$ as in Fig. 17.

\section{Conclusions}

We evaluated all partial decay widths corresponding to a two-body decay of the heavy MSSM Higgs bosons to scalar fermions, allowing for complex parameters. The decay modes are given in Eqs. (1) and (2). The evaluation is based on a full one-loop calculation of all decay channels, also including hard QED and QCD radiation. In the case of a discovery of additional Higgs bosons a subsequent precision measurement of their properties will be crucial to determine their nature and the underlying (SUSY) parameters. In order to yield a sufficient accuracy, one-loop corrections to the various Higgs-boson decay modes have to be considered. With the full one-loop calculation here presented for scalar fermions another step in the direction of a complete one-loop evaluation of all possible decay modes has been taken.

We first reviewed the one-loop renormalization procedure of the cMSSM, which is relevant for our calculation. In most cases we follow Ref. [57]. However, in the scalar sfermion sector, where we differ from Ref. [57] all relevant details are given.

We have discussed the calculation of the one-loop diagrams, the treatment of UV and IR divergences that are canceled by the inclusion of (hard and soft) QCD and QED radiation. We have checked our result against the literature (where loop corrections so far only for real parameters were 
Table 4 Thresholds in neutral Higgs-boson decays

\begin{tabular}{lll}
\hline TN1: & $M_{H^{ \pm}} \approx 598 \mathrm{GeV}$ & $m_{\tilde{\chi}_{1}^{0}}+m_{\tilde{\chi}_{1}^{0}}=m_{h_{2}} \approx 591 \mathrm{GeV}$ \\
TN2: & $M_{H^{ \pm}} \approx 624 \mathrm{GeV}$ & $m_{\tilde{\tau}_{2}}+m_{\tilde{\tau}_{2}}=m_{h_{2}}=618 \mathrm{GeV}$ \\
TN3: & $M_{H^{ \pm}} \approx 775 \mathrm{GeV}$ & $m_{\tilde{\chi}_{1}^{0}}+m_{\tilde{\chi}_{2}^{0}}=m_{h_{3}} \approx 771 \mathrm{GeV}$ \\
TN4: & $M_{H^{ \pm}} \approx 794 \mathrm{GeV}$ & $m_{\tilde{t}_{1}}+m_{\tilde{t}_{1}}=m_{h_{2}}=788 \mathrm{GeV}$ \\
TN5: & $M_{H^{ \pm}} \approx 805 \mathrm{GeV}$ & $m_{\tilde{\chi}_{1}^{0}}+m_{\tilde{\chi}_{3}^{0}}=m_{h_{2}} \approx 799 \mathrm{GeV}$ \\
TN6: & $M_{H^{ \pm}} \approx 948 \mathrm{GeV}$ & $m_{\tilde{\chi}_{1}^{ \pm}}+m_{\tilde{\chi}_{1}^{ \pm}}=m_{h_{3}} \approx 945 \mathrm{GeV}$ \\
TN7: & $M_{H^{ \pm}} \approx 954 \mathrm{GeV}$ & $m_{\tilde{\chi}_{2}^{0}}+m_{\tilde{\chi}_{2}^{0}}=m_{h_{3}} \approx 951 \mathrm{GeV}$ \\
TN8: & $M_{H^{ \pm}} \approx 982 \mathrm{GeV}$ & $m_{\tilde{\chi}_{2}^{0}}+m_{\tilde{\chi}_{3}^{0}}=m_{h_{2}} \approx 979 \mathrm{GeV}$ \\
TN9: & $M_{H^{ \pm}} \approx 1092 \mathrm{GeV}$ & $m_{\tilde{b}_{1}}+m_{\tilde{b}_{2}}=m_{h_{2}} \approx 1086 \mathrm{GeV}$ \\
TN10: & $M_{H^{ \pm}} \approx 1107 \mathrm{GeV}$ & $m_{\tilde{\chi}_{1}^{ \pm}}+m_{\tilde{\chi}_{2}^{ \pm}}=m_{h_{3}} \approx 1105 \mathrm{GeV}$ \\
TN11: & $M_{H^{ \pm}} \approx 1108 \mathrm{GeV}$ & $m_{\tilde{\chi}_{1}^{ \pm}}+m_{\tilde{\chi}_{2}^{ \pm}}=m_{h_{2}} \approx 1105 \mathrm{GeV}$ \\
TN12: & $M_{H^{ \pm}} \approx 1112 \mathrm{GeV}$ & $m_{\tilde{\chi}_{2}^{0}}+m_{\tilde{\chi}_{4}^{0}}=m_{h_{2}} \approx 1108 \mathrm{GeV}$ \\
TN13: & $M_{H^{ \pm}} \approx 1138 \mathrm{GeV}$ & $m_{\tilde{\chi}_{3}^{0}}+m_{\tilde{\chi}_{4}^{0}}=m_{h_{3}} \approx 1135 \mathrm{GeV}$ \\
TN14: & $M_{H^{ \pm}} \approx 1168 \mathrm{GeV}$ & $m_{\tilde{t}_{1}}+m_{\tilde{t}_{2}}=m_{h_{3}}=1165 \mathrm{GeV}$ \\
TN15: & $M_{H^{ \pm}} \approx 1171 \mathrm{GeV}$ & $m_{\tilde{t}_{1}}+m_{\tilde{t}_{2}}=m_{h_{2}}=1165 \mathrm{GeV}$ \\
TN16: & $M_{H^{ \pm}} \approx 1268 \mathrm{GeV}$ & $m_{\tilde{\chi}_{2}^{ \pm}}+m_{\tilde{\chi}_{2}^{ \pm}}=m_{h_{2}} \approx 1264 \mathrm{GeV}$ \\
TN17: & $M_{H^{ \pm}} \approx 1268 \mathrm{GeV}$ & $m_{\tilde{\chi}_{4}^{0}}+m_{\tilde{\chi}_{4}^{0}}=m_{h_{2}} \approx 1265 \mathrm{GeV}$ \\
TN18: & $M_{H^{ \pm}} \approx 1545 \mathrm{GeV}$ & $m_{\tilde{t}_{2}}+m_{\tilde{t}_{2}}=m_{h_{2}}=1542 \mathrm{GeV}$ \\
\hline
\end{tabular}

available) and in most cases found good agreement, once our set-up was changed to the one used in the existing analyses.

While the analytical calculation has been performed for all decay modes to sfermions, in the numerical analysis we concentrated on the decays to the third generation sfermions: scalar tops, bottoms, taus, and tau neutrinos. For the analysis we have chosen a parameter set that allows simultaneously a maximum number of two-body sfermionic decay modes. In the analysis either the charged Higgs-boson mass or the phase of a relevant trilinear coupling has been varied. For $M_{H^{ \pm}}$we investigated an interval starting at $M_{H^{ \pm}}=600 \mathrm{GeV}$ up to $M_{H^{ \pm}}=1.6 \mathrm{TeV}$, which roughly coincides with the reach of the LHC for high-luminosity running as well as an $e^{+} e^{-}$ collider with a center-of-mass energy up to $\sqrt{s} \sim 3 \mathrm{TeV}$.

In our numerical scenarios we compared the tree-level partial decay widths with the full one-loop corrected partial decay widths. In the case of decays to scalar quarks we also included for comparison the pure SQCD one-loop corrections. We concentrated on the analysis of the decay widths themselves, since the size of the corresponding branching ratios (and thus the size of the one-loop effects) is highly parameter dependent.

We found sizable, roughly $\mathcal{O}(15 \%)$, corrections in all the channels. The corrections tend to be larger for the decays to scalar quarks w.r.t. decays to scalar leptons. For some parts of the parameter space (not only close to thresholds) also larger corrections up to 30 or $40 \%$ (and in exceptional cases even higher) have been observed. Consequently, the full one-loop corrections should be taken into account for the interpretation of the searches for scalar fermions as well as for any future precision analyses of those decays.

The size of the tree-level decay widths and of the corresponding full one-loop corrections often depend strongly on the respective complex phase, i.e. $\varphi_{A_{t}}, \varphi_{A_{b}}$ or $\varphi_{A_{\tau}}$. The oneloop contributions often vary by a factor of $2-3$ as a function of the complex phases and sometimes can even turn negative. Neglecting the phase dependence could lead to a wrong impression of the relative size of the various decay widths. Furthermore, for certain values of the phases the relevant $(\propto \hat{\mathbf{Z}}$ matrix) real parts of specific couplings accidentally go through zero. Consequently, also the decay widths go to zero for these values, where the $\hat{\mathbf{Z}}$ matrix yields the dominating effect on the widths.

In case of decays to scalar quarks we have also compared with the pure SQCD result. We have found that in most cases the EW corrections are of similar size. Neglecting those can lead, depending on the parameter space, to a large over- or underestimate of the full one-loop corrections.

In the cases where a decay and its complex conjugate final state are possible we have evaluated both decay widths independently. The asymmetries, as a byproduct of our calculation, turn out to be sizable, in particular for decays into a pair of lighter and heavier scalar fermions.

The numerical results we have shown are, of course, dependent on the choice of the SUSY parameters. Nevertheless, they give an idea of the relevance of the full one-loop corrections. Decay channels (and their respective one-loop corrections) that may look unobservable due to the smallness of their decay width in our numerical examples could become important if other channels are kinematically forbidden. Following our analysis it is evident that the full oneloop corrections are mandatory for a precise prediction of the various branching ratios. We emphasize again that in many cases it is not sufficient to include only SQCD corrections, as electroweak corrections can be of comparable size. The full one-loop corrections should be taken into account in any precise determination of (SUSY) parameters from the decay of heavy MSSM Higgs bosons. The results for the heavy MSSM Higgs-boson decays will be implemented into the Fortran code FeynHiggs.

Acknowledgments We thank T. Hahn, W. Hollik, H. Rzehak, and G. Weiglein for helpful discussions. The work of S.H. is supported in part by CICYT (Grant FPA 2013-40715-P) and by the Spanish MICINN's Consolider-Ingenio 2010 Program under grant MultiDark CSD2009-00064.

Open Access This article is distributed under the terms of the Creative Commons Attribution 4.0 International License (http://creativecomm ons.org/licenses/by/4.0/), which permits unrestricted use, distribution, and reproduction in any medium, provided you give appropriate credit to the original author(s) and the source, provide a link to the Creative Commons license, and indicate if changes were made.

Funded by $\mathrm{SCOAP}^{3}$. 


\section{References}

1. H. Nilles, Phys. Rep. 110, 1 (1984)

2. R. Barbieri, Riv. Nuovo Cim. 11, 1 (1988)

3. H. Haber, G. Kane, Phys. Rep. 117, 75 (1985)

4. J. Gunion, H. Haber, Nucl. Phys. B 272, 1 (1986)

5. G. Aad et al. (ATLAS Collaboration), Phys. Lett. B 716, 1 (2012). arXiv:1207.7214 [hep-ex]

6. S. Chatrchyan et al. (CMS Collaboration), Phys. Lett. B 716, 30 (2012). arXiv:1207.7235 [hep-ex]

7. G. Aad et al. (ATLAS Collaboration), JHEP 1411, 056 (2014). arXiv: 1409.6064 [hep-ex]

8. V. Khachatryan et al. (CMS Collaboration), JHEP 1410, 160 (2014). arXiv:1408.3316 [hep-ex]

9. A. Holzner (ATLAS and CMS Collaborations), arXiv:1411.0322 [hep-ex]

10. H. Baer et al., The international linear collider technical design report-volume 2: Physics, arXiv:1306.6352 [hep-ph]

11. TESLA Technical Design Report (TESLA Collaboration) Part 3, Physics at an $e^{+} e^{-}$linear collider. arXiv:hep-ph/0106315. See http://www.tesla.desy.de/new_pages/TDR_CD/start.html

12. K. Ackermann et al., DESY-PROC-2004-01

13. J. Brau et al. (ILC Collaboration), ILC reference design report volume 1-executive summary. arXiv:0712.1950 [physics.acc-ph]

14. G. Aarons et al. (ILC Collaboration), International linear collider reference design report volume 2: physics at the ILC. arXiv:0709.1893 [hep-ph]

15. L. Linssen, A. Miyamoto, M. Stanitzki, H. Weerts, arXiv:1202.5940 [physics.ins-det]

16. H. Abramowicz et al. (CLIC Detector and Physics Study Collaboration), Physics at the CLIC $e^{+} e^{-}$linear collider-input to the snowmass process 2013. arXiv:1307.5288 [hep-ex]

17. G. Weiglein et al. (LHC/ILC Study Group), Phys. Rep. 426, 47 (2006). arXiv:hep-ph/0410364

18. A. De Roeck et al., Eur. Phys. J. C 66, 525 (2010). arXiv:0909.3240 [hep-ph]

19. A. De Roeck, J. Ellis, S. Heinemeyer, CERN Cour. 49N10, 27 (2009)

20. K. Williams, H. Rzehak, G. Weiglein, Eur. Phys. J. C 71, 1669 (2011). arXiv:1103.1335 [hep-ph]

21. S. Heinemeyer, W. Hollik, G. Weiglein, Eur. Phys. J. C 16, 139 (2000). arXiv:hep-ph/0003022

22. D. Noth, M. Spira, Phys. Rev. Lett. 101, 181801 (2008). arXiv:0808.0087 [hep-ph]

23. D. Noth, M. Spira, JHEP 1106, 084 (2011). arXiv:1001.1935 [hep-ph]

24. L. Mihaila, C. Reisser, JHEP 1008, 021 (2010). arXiv:1007.0693 [hep-ph]

25. R. Hempfling, Phys. Rev. D 49, 6168 (1994)

26. L. Hall, R. Rattazzi, U. Sarid, Phys. Rev. D 50, 7048 (1994). arXiv:hep-ph/9306309

27. M. Carena, M. Olechowski, S. Pokorski, C. Wagner, Nucl. Phys. B 426, 269 (1994). arXiv:hep-ph/9402253

28. M. Carena, D. Garcia, U. Nierste, C. Wagner, Nucl. Phys. B 577, 577 (2000). arXiv:hep-ph/9912516

29. S. Heinemeyer, W. Hollik, Nucl. Phys. B 474, 32 (1996). arXiv:hep-ph/9602318

30. A. Bredenstein, A. Denner, S. Dittmaier, M. Weber, Phys. Rev. D 74, 013004 (2006). arXiv:hep-ph/0604011

31. A. Bredenstein, A. Denner, S. Dittmaier, M. Weber, JHEP 0702, 080 (2007). arXiv:hep-ph/0611234

32. A. Bredenstein, A. Denner, S. Dittmaier, A. Mück, M. Weber, see http://omnibus.uni-freiburg.de/ ^sd565/programs/prophecy4f/ prophecy4f.html
33. M. Frank, T. Hahn, S. Heinemeyer, W. Hollik, H. Rzehak, G. Weiglein, JHEP 0702, 047 (2007). arXiv:hep-ph/0611326

34. S. Heinemeyer, W. Hollik, G. Weiglein, Eur. Phys. J. C 9, 343 (1999). arXiv:hep-ph/9812472

35. S. Heinemeyer, W. Hollik, G. Weiglein, Comput. Phys. Commun. 124, 76 (2000). arXiv:hep-ph/9812320

36. T. Hahn, S. Heinemeyer, W. Hollik, H. Rzehak, G. Weiglein, Comput. Phys. Commun. 180, 1426 (2009). See www.feynhiggs.de

37. G. Degrassi, S. Heinemeyer, W. Hollik, P. Slavich, G. Weiglein, Eur. Phys. J. C 28, 133 (2003). arXiv:hep-ph/0212020

38. T. Hahn, S. Heinemeyer, W. Hollik, H. Rzehak, G. Weiglein, Phys. Rev. Lett. 112, 141801 (2014). arXiv:1312.4937 [hep-ph]

39. A. Djouadi, J. Kalinowsli, M. Spira, Comput. Phys. Commun. 108, 56 (1998). arXiv:hep-ph/9704448

40. M. Spira, Fortschr. Phys. 46, 203 (1998). arXiv:hep-ph/9705337

41. A. Djouadi, J. Kalinowski, M. Mühlleitner, M. Spira, arXiv: 1003.1643 [hep-ph]

42. S. Heinemeyer et al. (LHC Higgs Cross Section Working Group), arXiv:1307.1347 [hep-ph]

43. M. Carena, S. Heinemeyer, O. Stål, C. Wagner, G. Weiglein, Eur. Phys. J. C 73, 2552 (2013). arXiv:1302.7033 [hep-ph]

44. A. Bartl, H. Eberl, K. Hidaka, T. Kon, W. Majerotto, Y. Yamada, Phys. Lett. B 373, 117 (1996). arXiv:hep-ph/9508283

45. A. Bartl, H. Eberl, K. Hidaka, T. Kon, W. Majerotto, Y. Yamada, Phys. Lett. B 402, 303 (1997). arXiv:hep-ph/9701398

46. H. Eberl, K. Hidaka, S. Kraml, W. Majerotto, Y. Yamada, Phys. Rev. D 62, 055006 (2000). arXiv:hep-ph/9912463

47. C. Weber, H. Eberl, W. Majerotto, Phys. Lett. B 572, 56 (2003). arXiv:hep-ph/0305250

48. C. Weber, H. Eberl, W. Majerotto, Phys. Rev. D 68, 093011 (2003). arXiv:hep-ph/0308146

49. C. Weber, K. Kovarik, H. Eberl, W. Majerotto, Nucl. Phys. B 776, 138 (2007). arXiv:hep-ph/0701134

50. W. Frisch, H. Eberl, H. Hluchá, Comput. Phys. Commun. 182, 2219 (2011). arXiv:1012.5025 [hep-ph]

51. S. Heinemeyer, H. Rzehak, C. Schappacher, Phys. Rev. D 82, 075010 (2010). arXiv:1007.0689 [hep-ph]

52. S. Heinemeyer, H. Rzehak, C. Schappacher, PoSCHARGED 2010, 039 (2010). arXiv:1012.4572 [hep-ph]

53. T. Fritzsche, S. Heinemeyer, H. Rzehak, C. Schappacher, Phys. Rev. D 86, 035014 (2012). arXiv: 1111.7289 [hep-ph]

54. S. Heinemeyer, C. Schappacher, Eur. Phys. J. C 72, 2136 (2012). arXiv: 1204.4001 [hep-ph]

55. A. Arhrib, A. Djouadi, W. Hollik, C. Jünger, Phys. Rev. D 57, 5860 (1998). arXiv:hep-ph/9702426

56. E. Accomando, G. Chachamis, F. Fugel, M. Spira, M. Walser, Phys. Rev. D 85, 015004 (2012). arXiv:1103.4283 [hep-ph]

57. T. Fritzsche, T. Hahn, S. Heinemeyer, F. von der Pahlen, H. Rzehak, C. Schappacher, Comput. Phys. Commun. 185, 1529 (2014). arXiv:1309.1692 [hep-ph]

58. S. Heinemeyer, W. Hollik, H. Rzehak, G. Weiglein, Phys. Lett. B 652, 300 (2007). arXiv:0705.0746 [hep-ph]

59. H. Rzehak, PhD thesis: Two-loop contributions in the supersymmetric Higgs sector. Technische Universität München, 2005. See http://www.nbn-resolving.de/ with URN: nbn:de:bvb:91diss20050923-0853568146

60. A. Bartl, H. Eberl, K. Hidaka, S. Kraml, W. Majerotto, W. Porod, Y. Yamada, Phys. Lett. B 419, 243 (1998). arXiv:hep-ph/9710286

61. A. Bartl, H. Eberl, K. Hidaka, S. Kraml, W. Majerotto, W. Porod, Y. Yamada, Phys. Rev. D 59, 115007 (1999). arXiv:hep-ph/9806299

62. A. Djouadi, P. Gambino, S. Heinemeyer, W. Hollik, C. Jünger, G. Weiglein, Phys. Rev. Lett. 78, 3626 (1997). arXiv:hep-ph/9612363 
63. A. Djouadi, P. Gambino, S. Heinemeyer, W. Hollik, C. Jünger, G. Weiglein, Phys. Rev. D 57, 4179 (1998). arXiv:hep-ph/9710438

64. W. Hollik, H. Rzehak, Eur. Phys. J. C 32, 127 (2003). arXiv:hep-ph/0305328

65. S. Heinemeyer, W. Hollik, H. Rzehak, G. Weiglein, Eur. Phys. J. C 39, 465 (2005). arXiv:hep-ph/0411114

66. J. Beringer et al. (Particle Data Group), Phys. Rev. D 86 (2012) 010001 and 2013 partial update for the 2014 edition

67. K. Chetyrkin, J. Kühn, M. Steinhauser, Comput. Phys. Commun. 133, 43 (2000). arXiv:hep-ph/0004189

68. B. Schmidt, M. Steinhauser, Comput. Phys. Commun. 183, 1845 (2012). arXiv:1201.6149 [hep-ph]

69. M. Carena, D. Garcia, U. Nierste, C. Wagner, Nucl. Phys. B 577, 577 (2000). arXiv:hep-ph/9912516

70. L. Hall, R. Rattazzi, U. Sarid, Phys. Rev. D 50, 7048 (1994). arXiv:hep-ph/9306309

71. M. Carena, M. Olechowski, S. Pokorski, C. Wagner, Nucl. Phys. B 426, 269 (1994). arXiv:hep-ph/9402253

72. M. Carena, J. Ellis, A. Pilaftsis, C. Wagner, Nucl. Phys. B 586, 92 (2000). arXiv:hep-ph/0003180

73. J. Guasch, P. Hafliger, M. Spira, Phys. Rev. D 68, 16 (2003). arXiv:hep-ph/0305101

74. R. Harlander, L. Mihaila, M. Steinhauser, Phys. Rev. D 72, 095009 (2005). arXiv:hep-ph/0509048

75. R. Harlander, L. Mihaila, M. Steinhauser, Phys. Rev. D 76, 055002 (2007). arXiv:0706.2953 [hep-ph]

76. A. Denner, S. Dittmaier, M. Roth, D. Wackeroth, Nucl. Phys. B 560, 33 (1999). arXiv:hep-ph/9904472

77. J. Küblbeck, M. Böhm, A. Denner, Comput. Phys. Commun. 60, 165 (1990)

78. T. Hahn, Comput. Phys. Commun. 140, 418 (2001). arXiv:hep-ph/0012260

79. T. Hahn, C. Schappacher, Comput. Phys. Commun. 143, 54 (2002). hep-ph/0105349. Program, user's guide and model files are available via www.feynarts.de

80. T. Hahn, M. Pérez-Victoria, Comput. Phys. Commun. 118, 153 (1999). arXiv:hep-ph/9807565

81. F. del Aguila, A. Culatti, R. Muñoz Tapia, M. Pérez-Victoria, Nucl. Phys. B 537, 561 (1999). arXiv:hep-ph/9806451

82. W. Siegel, Phys. Lett. B 84, 193 (1979)

83. D. Capper, D. Jones, P. van Nieuwenhuizen, Nucl. Phys. B 167, 479 (1980)

84. D. Stöckinger, JHEP 0503, 076 (2005). arXiv:hep-ph/0503129

85. W. Hollik, D. Stöckinger, Phys. Lett. B 634, 63 (2006). arXiv:hep-ph/0509298

86. A. Denner, Fortsch. Phys. 41, 307 (1993). arXiv:0709.1075 [hep$\mathrm{ph}]$

87. The couplings can be found in HMix.ps.gz as part of the FeynArts package [58]
88. A. Arhrib, Private communication, 08.06.2014

89. J. Aguilar-Saavedra et al., Eur. Phys. J. C 46, 43 (2006). arXiv:hep-ph/0511344

90. J. Frère, D. Jones, S. Raby, Nucl. Phys. B 222, 11 (1983)

91. M. Claudson, L. Hall, I. Hinchliffe, Nucl. Phys. B 228, 501 (1983)

92. C. Kounnas, A. Lahanas, D. Nanopoulos, M. Quiros, Nucl. Phys. B 236, 438 (1984)

93. J. Gunion, H. Haber, M. Sher, Nucl. Phys. B 306, 1 (1988)

94. J. Casas, A. Lleyda, C. Munoz, Nucl. Phys. B 471, 3 (1996). arXiv:hep-ph/9507294

95. P. Langacker, N. Polonsky, Phys. Rev. D 50, 2199 (1994). arXiv:hep-ph/9403306

96. A. Strumia, Nucl. Phys. B 482, 24 (1996). arXiv:hep-ph/9604417

97. S. Dimopoulos, S. Thomas, Nucl. Phys. B 465, 23 (1996). arXiv:hep-ph/9510220

98. M. Dugan, B. Grinstein, L. Hall, Nucl. Phys. B 255, 413 (1985)

99. D. Demir, O. Lebedev, K. Olive, M. Pospelov, A. Ritz, Nucl. Phys. B 680, 339 (2004). arXiv:hep-ph/0311314

100. D. Chang, W. Keung, A. Pilaftsis, Phys. Rev. Lett. 82, 900 (1999). hep-ph/9811202 [Erratum-ibid. 83, 3972 (1999)]

101. A. Pilaftsis, Phys. Lett. B 471, 174 (1999). arXiv:hep-ph/9909485

102. O. Lebedev, K. Olive, M. Pospelov, A. Ritz, Phys. Rev. D 70, 016003 (2004). arXiv:hep-ph/0402023

103. W. Hollik, J. Illana, S. Rigolin, D. Stöckinger, Phys. Lett. B 416, 345 (1998). arXiv:hep-ph/9707437

104. W. Hollik, J. Illana, S. Rigolin, D. Stöckinger, Phys. Lett. B 425, 322 (1998). arXiv:hep-ph/9711322

105. P. Nath, Phys. Rev. Lett. 66, 2565 (1991)

106. Y. Kizukuri, N. Oshimo, Phys. Rev. D 46, 3025 (1992)

107. T. Ibrahim, P. Nath, Phys. Lett. B 418, 98 (1998). arXiv:hep-ph/9707409

108. T. Ibrahim, P. Nath, Phys. Rev. D 57, 478 (1998). arXiv:hep-ph/9708456 [Erratum-ibid. D 58, 019901 (1998); Erratum-ibid. D 60, 079903 (1998); Erratum-ibid. D 60, 119901 (1999)]

109. M. Brhlik, G. Good, G. Kane, Phys. Rev. D 59, 115004 (1999). arXiv:hep-ph/9810457

110. S. Abel, S. Khalil, O. Lebedev, Nucl. Phys. B 606, 151 (2001). arXiv:hep-ph/0103320

111. Y. Li, S. Profumo, M. Ramsey-Musolf, JHEP 1008, 062 (2010). arXiv:1006.1440 [hep-ph]

112. V. Barger, T. Falk, T. Han, J. Jiang, T. Li, T. Plehn, Phys. Rev. D 64, 056007 (2001). arXiv:hep-ph/0101106

113. H. Heath, C. Lynch, S. Moretti, C. Shepherd-Themistocleous, arXiv:0901.1676 [hep-ph]

114. A. Datta, A. Djouadi, M. Guchait, F. Moortgat, Nucl. Phys. B 681, 31 (2004). arXiv:hep-ph/0303095

115. G. 't Hooft, M. Veltman, Nucl. Phys. B 153, 365 (1979) 